\title{
Existence, uniqueness and ergodic properties for time- homogeneous Itô-SDEs with locally integrable drifts and Sobolev diffusion coefficients 1
}

\author{
Haesung Lee, Gerald Trutnau
}

\begin{abstract}
Using elliptic and parabolic regularity results in $L^{p}$-spaces and generalized Dirichlet form theory, we construct for every starting point weak solutions to SDEs in $\mathbb{R}^{d}$ up to their explosion times including the following conditions. For arbitrary but fixed $p>d$ the diffusion coefficient $A=\left(a_{i j}\right)_{1 \leq i, j \leq d}$ is locally uniformly strictly elliptic with functions $a_{i j} \in H_{l o c}^{1, p}\left(\mathbb{R}^{d}\right)$ and the drift coefficient $\mathbf{G}=\left(g_{1}, \ldots, g_{d}\right)$ consists of functions $g_{i} \in L_{l o c}^{p}\left(\mathbb{R}^{d}\right)$. The solution originates by construction from a Hunt process with continuous sample paths on the one-point compactification of $\mathbb{R}^{d}$ and the corresponding SDE is by a known local well-posedness result pathwise unique up to an explosion time. Just under the given assumptions we show irreducibility and the strong Feller property on $L^{1}\left(\mathbb{R}^{d}, m\right)+L^{\infty}\left(\mathbb{R}^{d}, m\right)$ of its transition function, and the strong Feller property on $L^{q}\left(\mathbb{R}^{d}, m\right)+L^{\infty}\left(\mathbb{R}^{d}, m\right), q=\frac{d p}{d+p} \in(d / 2, p / 2)$, of its resolvent, which both include the classical strong Feller property. We present moment inequalities and classical-like non-explosion criteria for the solution which lead to pathwise uniqueness results up to infinity under presumably optimal general non-explosion conditions. We further present explicit conditions for recurrence and ergodicity, including existence as well as uniqueness of invariant probability measures.
\end{abstract}

Mathematics Subject Classification (2010): primary; 60H20, 47D07, 60J35; secondary: 31C25, 60J60, 35B65.

Keywords: pathwise uniqueness, non-explosion, recurrence, ergodicity, invariant probability measure, strong Feller property, elliptic and parabolic regularity.

\section{Introduction}

Consider the stochastic differential equation (SDE)

$$
X_{t}=x_{0}+\int_{0}^{t} \sigma\left(X_{s}\right) d W_{s}+\int_{0}^{t} \mathbf{G}\left(X_{s}\right) d s, 0 \leq t<\zeta, x_{0} \in \mathbb{R}^{d}
$$

where $W=\left(W^{1}, \ldots, W^{m}\right)$ is a standard $m$-dimensional Brownian motion starting from zero, $A=\left(a_{i j}\right)_{1 \leq i, j \leq d}=\sigma \sigma^{T}, \sigma=\left(\sigma_{i j}\right)_{1 \leq i \leq d, 1 \leq j \leq m}$ and $\mathbf{G}=\left(g_{1}, \ldots, g_{d}\right)$ are measurable and

$$
\zeta:=\inf \left\{t \geq 0: X_{t} \notin \mathbb{R}^{d}\right\}=\lim _{n \rightarrow \infty} \inf \left\{t \geq 0: X_{t} \notin B_{n}\right\}
$$

is the explosion time (or life time) of $X$, i.e. the time when $X$ has left any open Euclidean ball $B_{n}$ of radius $n$ about the origin. By a classical result, if $\sigma, \mathbf{G}$ consist of locally Lipschitz

\footnotetext{
${ }^{1}$ This research was supported by Basic Science Research Program through the National Research Foundation of Korea(NRF) funded by the Ministry of Education(NRF-2017R1D1A1B03035632).
} 
continuous functions and satisfy a linear growth condition, then (1) with $\zeta=\infty$ has a pathwise unique solution that is strong, i.e. adapted to the filtration generated by $W$ ([20, IV. Theorems 2.4 and 3.1]). Note that the just mentioned reference and most of those below also treat the time inhomogeneous case but we only discuss results in the time homogeneous case, i.e. results related to (1).

We call a solution that is pathwise unique and strong up to $\zeta(\zeta$ being possibly finite, cf. [20, IV. Definition 2.1]) strongly unique up to $\zeta$. Strong uniqueness results for (11) with $\zeta=\infty$ for only measurable coefficients were given starting from [40], [35], [36]. In these works $\sigma$ is nondegenerate and $\sigma, \mathbf{G}$ are bounded. Regarding bounded coefficients one can also mention the later work [3]. To our knowledge the first strong uniqueness results for unbounded measurable coefficients start with [18, Theorem 2.1], while weak existence results appeared to exist earlier (cf. introduction of [18]). In [18, Theorem 2.1] $\sigma$ may be chosen locally Lipschitz, with $\sigma \sigma^{T}$ globally uniformly strictly elliptic and $g_{i} \in L_{l o c}^{2(d+1)}\left(\mathbb{R}^{d}\right)$ with the following growth condition to ensure non-explosion ([18, Assumption 2.1]): there exists a constant $M \geq 0$ and a non-negative function $F \in L^{d+1}\left(\mathbb{R}^{d}\right)$ such that almost everywhere

$$
\|\mathbf{G}\|=\left(\sum_{i=1}^{d} g_{i}^{2}\right)^{1 / 2} \leq M+F
$$

Note that this growth condition does not allow for linear growth and that it depends only on almost every point, which is natural since integrals such as the one in (11) involving $\mathbf{G}$ should not depend on the particular Borel version chosen for G. In [37], the following result was obtained: if $\sigma$ consists of continuous functions and is globally uniformly non-degenerate, i.e. $A(x) \geq C \cdot \operatorname{Id}$ in the quadratic form sense for some constant $C>0$ and every $x \in \mathbb{R}^{d}$ and $g_{i}, \partial_{k} \sigma_{i j} \in L_{l o c}^{2(d+1)}\left(\mathbb{R}^{d}\right)$ for any $i, j, k$, then (11) has a strongly unique solution up to its explosion time. In [37, Theorem 1.1(i) and (ii)] two non-explosion conditions are given. Both require the global boundedness of $\sigma$ and then only depend on $\mathbf{G}$. The first one is similar to the one of [18] given above. The second one is as follows: there exist a constant $M \geq 0$, and vector fields $\mathbf{H}, \mathbf{F}_{i}$, with $\left\|\mathbf{F}_{i}\right\| \in L^{p_{i}}\left(\mathbb{R}^{d}\right)$, $p_{i} \geq 2(d+1)$, such that almost everywhere

$$
\mathbf{G}=\sum_{i=1}^{k} \mathbf{F}_{i}+\mathbf{H} \quad \text { with } \quad\|\mathbf{H}(x)\| \leq M\left(1+1_{\{\|x\|>e\}}\|x\| \log \|x\|\right) .
$$

This non-explosion condition allows for linear growth and can cover singularities of $\mathbf{G}$, a phenomenon that can not occur for SDEs with continuous coefficients, since these are of course locally bounded. Prior to [37, the following was obtained in [21]: if $\sigma$ is the identity matrix, so that the local martingale part in (11) is just a $d$-dimensional Brownian motion $W=\left(W^{1}, \ldots, W^{d}\right)$ and $g_{i} \in L_{l o c}^{p}\left(\mathbb{R}^{d}\right), 1 \leq i \leq d$, for some $p>d$, with

$$
\int_{0}^{t}\left\|\mathbf{G}\left(X_{s}\right)\right\|^{r} d s<\infty \mathbb{P}_{x_{0}} \text {-almost surely on }\{t<\zeta\}
$$

where $r=2$ and $\mathbb{P}_{x_{0}}$ is the distribution on the paths starting form $x_{0}$, then (11) has a strongly unique solution up to its explosion time. Besides a global $L_{q^{-}} L_{p^{-}}$-condition which does not allow for linear growth a rather special and not really explicit non-explosion condition is presented in [21]. Its formulation is quite long but roughly one can say it is given by assuming that $\mathbf{G}$ is the weak gradient of a function $\psi$ which is a kind of Lyapunov function for (1). For the precise 
statement, we refer to [21, Assumption 2.1]. The strong uniqueness result of [21] was generalized among others in [38, Theorem 1.3] to the case of non-trivial continuous $d \times d$-dispersion matrix $\sigma$ with corresponding locally uniformly strictly elliptic diffusion matrix and $\sigma_{i j} \in H_{l o c}^{1, p}\left(\mathbb{R}^{d}\right)$ where $p>d$ is the same as for $\mathbf{G}$, relaxing condition (2) to the natural one, i.e. $r=1$ (see also Remark [3.7(i)) but no non-explosion condition related to the local conditions of [38, Theorem 1.3] is given. Only a global $L_{p}$-integrability condition in space is given in [38, Theorem 1.2], which again does not allow for linear growth. Note that [38, Theorem 1.3] also holds under the conditions of Remark 3.3(ii) and that we can handle this case but disregard it for the reasons mentioned in Remark 3.3. Conditions for non-explosion, as well as irreducibility and strong Feller properties that we will discuss below, were given in [39, Theorems 1.2 and 1.7]. The conditions are formulated for the general time-dependent case but seem to be not optimal when restricted to the time-homogeneous case. For instance, growth conditions are formulated separately, first for locally bounded drift coefficient, then for locally unbounded drift coefficient $\mathbf{G}$, whereas our results show that this is unnecessary in the time-homogeneous case. Moreover the growth conditions for possibly locally unbounded drift coefficient in [39, Theorem 1.7] require local integrability of order $p>2 d+2$, global ellipticity, Lipschitz continuity outside a ball, i.e. outside a neighborhood of the singularity, and the norm of the drift needs to satisfy a linear growth condition, but as we will see below, we do not need any of these conditions. On the other hand, weak differentiability of the solution as well as corresponding moment inequalities for the weak gradient of the solution are presented in [39, Theorems 1.2 and 1.7], which we both do not consider. For more discussion on the results of [39] when restricted to the time-homogeneous case see below and Remark 4.19 below.

The strong uniqueness results of [21] were also recovered in [13] using a different method of proof which allowed to obtain additional insight on the solution. For instance, the $\alpha$-Hölder continuity of the solution for arbitrary $\alpha \in(0,1)$ and the differentiability in $L^{2}\left(\Omega \times[0, T], \mathbb{R}^{d}\right)$ (here $\Omega$ is the path space) with respect to the initial condition. For the latter result see [14].

Now we will describe our results and compare them with previous works. Let $p>d$ be arbitrary but fixed. For $A=\left(a_{i j}\right)_{1 \leq i, j \leq d}$ and $\mathbf{G}$ satisfying our basic assumptions, i.e. $a_{i j} \in$ $H_{l o c}^{1, p}\left(\mathbb{R}^{d}\right), 1 \leq i, j \leq d$, such that $A$ is locally uniformly strictly elliptic (cf. (4) below), and $\mathbf{G}=\left(g_{1}, \ldots, g_{d}\right) \in L_{l o c}^{p}\left(\mathbb{R}^{d}, \mathbb{R}^{d}\right)$, we construct a weak solution to (1) up to $\zeta$ using elliptic and parabolic regularity results and generalized Dirichlet form techniques. This is achieved in Theorem 3.19 and Remark 3.20 and $\sigma$ can be chosen as in Theorem 3.19)(i) or (ii). The weak solution originates by construction from a Hunt process with continuous sample paths on the one-point compactification of $\mathbb{R}^{d}$ (see Theorem 3.12). Moreover, the combination of bilinear form and PDE techniques allows us just under the above assumptions to obtain the $L^{[1, \infty]}\left(\mathbb{R}^{d}, m\right)$-strong Feller property of the transition function of the weak solution, and the $L^{[q, \infty]}\left(\mathbb{R}^{d}, m\right)$-strong Feller property, $q=\frac{d p}{d+p} \in(d / 2, p / 2)$, of its resolvent, which both include the classical strong Feller property (cf. Theorem [3.8, Proposition 3.10 and Lemma 3.14). Additionally, the Hunt process is irreducible in the probabilistic sense and the semigroup of the underlying generalized Dirichlet form is strictly irreducible (cf. Corollary 4.8). Using the facts that we obtained from the construction method, the solution can be shown to be non-explosive, if there exists a constant $M>0$ and some $N_{0} \in \mathbb{N}$, such that

$$
-\frac{\langle A(x) x, x\rangle}{\|x\|^{2}+1}+\frac{1}{2} \operatorname{trace} A(x)+\langle\mathbf{G}(x), x\rangle \leq M\left(\|x\|^{2}+1\right)\left(\ln \left(\|x\|^{2}+1\right)+1\right)
$$

for a.e. $x \in \mathbb{R}^{d} \backslash B_{N_{0}}$. This is proven in Theorem 4.2 using supermartingales. The conditions allow for linear growth, for locally unbounded drifts and an interplay between diffusion and 
drift coefficients such that (even outside $B_{N_{0}}$ ) superlinear growth of $\mathbf{G}$ is possible if $\langle\mathbf{G}(x), x\rangle$ is non-positive and superlinear growth of $\mathbf{G}$ and $A$ is possible if diffusion and drift coefficients compensate each other.

Once we have constructed a weak solution up to its explosion time, we concentrate on nonexplosion conditions for it. Under a non-explosion condition or more generally under the assumption of non-explosion of the weak solution, it is a global weak solution, whose corrseponding SDE is strongly unique up to $\infty$ by [38, 21. This observation was first employed in [27] and leads to new non-explosion results for the pathwise unique local solutions in [38, 21]. As application of this observation, we obtain strong uniqueness of (1) up to $\infty$ just under the additional non-explosion condition (3) (or more generally under any condition that guarantees the non-explosion of our weak solution, see our main Theorem 5.1). But we obtain more than this. Namely, the pathwise unique solution $\left(X_{t}\right)_{t \geq 0}$ in Theorem 5.1 is not only strong but satisfies all previously derived properties. Our strong Feller property results generalize the ones obtained in [1, Propositions 3.2 and 3.8] and [5, Theorem 2.8] and improve the results related to the timehomogeneous case in [39]. There $\mathbb{M}$ should be non-explosive to obtain merely the classical strong Feller property (cf. also Remark 3.9(iii)). Also, the irreducibility (in the probabilistic sense) here is just obtained under the mentioned basic assumptions on $A$ and $\mathbf{G}$, whereas the assumptions to obtain irreducibility in [39] appear to be quite involved. Additionally, our method provides implicitly a candidate for an invariant measure as well as for a stationary distribution and we derive several explicit sufficient conditions for recurrence and ergodicity, including existence and uniqueness of invariant probability measures (see Section 4.2). Moreover, we derive moment inequalities for the solution (see Theorem 4.4 which complements [13, Proposition 14] and [22, Lemma 3.2 of Section 2.3, Theorem 4.1 of Section 2.4]). All these are advantages over the methods that were previously employed in [18, [21, 38, [39], [13, and we are able to generalize and even improve many of the classical results in the time-homogeneous case for locally bounded coefficients (see [4] and the standard reference [25]) to the case of locally unbounded coefficients (see for instance Remark 4.7 and Theorem 4.13). Here we should emphasize that in contrast to previous literature where Krylov's estimate is used as a main technique, the only time where we indirectly use Krylov's estimate is when we apply the local pathwise uniqueness result of [38, Theorem 1.3] (see our main Theorem [5.1). For all other properties that we prove, we use the method that we described above.

The paper is organized as follows. In Section 2 we introduce the notations that are used throughout the text. In Section 3 we develop the analysis to define rigorously the infinitesimal generator $L$ that a solution to (11) should have under our assumptions. We first use a result of [30], i.e. that a strongly continuous semigroup of contractions and a generalized Dirichlet form on some $L^{2}$ space associated to an extension of $L$ as in (17) below, can be constructed. For this construction, one needs some weak divergence free property of the anti-symmetric part of the drift. Theorem 3.2 (from [7, Theorem 2.4.1]) implies that one can obtain this property with respect to a measure $m=\rho d x$, where $\rho$ is some strictly positive continuous function, under our basic assumptions on $A=\left(a_{i j}\right)_{1 \leq i, j \leq d}$ and $\mathbf{G}$. Typically, the density $\rho$ is not explicit and not a probability density but has the regularity $\rho \in H_{l o c}^{1, p}\left(\mathbb{R}^{d}\right)$. In the whole article we just use its existence as a tool and do not need to know its explicit form, except in Section 4.1.2 and parts of Section 4.2, see Theorem 4.11 there. Subsequently, we use the elliptic regularity result Proposition 3.4 (from [5, Theorem 5.1]) and our parabolic regularity result Theorem 3.8 which we derive from results in [2] to obtain the regularity as stated in Proposition 3.10 and $(\mathbf{H 2})^{\prime}$. Following the basic idea from [1], we may then use the Dirichlet form method to obtain the existence of a Hunt process $\mathbb{M}$ with transition function $\left(P_{t}\right)_{t>0}$ associated to the mentioned extension of $L$, with continuous 
sample paths on the one point compactification $\mathbb{R}_{\Delta}^{d}$ of $\mathbb{R}^{d}$ with $\Delta$ (see Theorem 3.12 ). To obtain its existence we crucially make use of the existence of such a Hunt process for merely almost every starting point which we obtain from [34, 33]. Once $\mathbb{M}$ is constructed, we can use standard methods from [20] (see Theorem 3.19 and Remark 3.20) to arrive at the identification of a weak solution to (1) up to $\zeta$. In Section 4 , we first develop non-explosion criteria for $\mathbb{M}$. The first such statement is obtained in Theorem 4.2 by some probabilistic technique using supermartingales which dates back at least to [31, 10.2] (see also [12, Chapter 5.3] and [25, Section 6.7]). The statement is basically that there exists a strictly positive $C^{2}$-function on $\mathbb{R}^{d}$ with nice growth properties at infinity such that $M u-L u \geq 0$ a.e. for some constant $M>0$. In the case of an analytic proof it seems to go back to [19] (see [11, Theorem 2.4]). Using the strong Feller property, the non-explosion conditions of Theorem 4.2 can also be recovered from [30, as explained in Remark 4.3. In Section 4.2 we discuss recurrence and other ergodic properties involving and not involving the density $\rho$. As previously mentioned, $\rho$ is usually not explicit but can be assumed to be explicit (if needed) as explained in Remark 4.5. Using a Harnack inequality from [2], we then show that the semigroup of the underlying generalized Dirichlet form is strictly irreducible in Corollary 4.8(i). Consequently, we can apply explicit volume growth conditions from [17] to obtain recurrence (cf. Theorem 4.11). In the general case, when $\rho$ is not explicitly known, we can also derive explicit recurrence criteria. Theorem 4.13, that is applicable just under our basic assumptions on $A=\left(a_{i j}\right)_{1 \leq i, j \leq d}$ and $\mathbf{G}$, generalizes [25, Chapter 6, Theorem 1.2] which assumes the drift to be locally bounded. Moreover the proof of Theorem 4.13 is different from the one of [25. Chapter 6, Theorem 1.2] and uses basic results of [17], as well as strict irreducibility from Corollary 4.8(i) and Proposition 4.9, In Proposition 4.17, we derive again just under our basic assumptions on $A$ and $\mathbf{G}$ an explicit criterion for ergodicity of $\mathbb{M}$, including the existence of a unique invariant probability measure. Section 5 is devoted to the mentioned application to pathwise uniqueness results.

\section{Notations}

Throughout, we consider the Euclidean space $\mathbb{R}^{d}, d \geq 2$, equipped with the Euclidean inner product $\langle\cdot, \cdot\rangle$, the Euclidean norm $\|\cdot\|$ and the Borel $\sigma$-algebra $\mathcal{B}\left(\mathbb{R}^{d}\right)$. We write $|\cdot|$ for the absolute value in $\mathbb{R}$. For $r \in \mathbb{R}, r>0$ and $x \in \mathbb{R}^{d}$, let $B_{r}(x):=\left\{y \in \mathbb{R}^{d}:\|x-y\|<r\right\}$ and denote its closure by $\bar{B}_{r}(x)$ (similarly for a subset $A \subset \mathbb{R}^{d}$, let $\bar{A}$ denote its closure). If $x=0$, we simply write $B_{r}$ and $\bar{B}_{r}$. We call a subset $B \subset \mathbb{R}^{d}$, for which $B=B_{r}(x)$ for some $r>0$ and $x \in \mathbb{R}^{d}$, a ball. Let $R_{x}(r)$ denote the open cube in $\mathbb{R}^{d}$ with edge length $r>0$ and center $x \in \mathbb{R}^{d}$ and denote its closure by $\bar{R}_{x}(r)$. The minimum of two values $a$ and $b$ is denoted by $a \wedge b:=\min (a, b)$ and the maximum is denoted by $a \vee b:=\max (a, b)$. For two sets $A, B$, we define $A+B:=\{a+b: a \in A$ and $b \in B\}$.

The set of all $\mathcal{B}\left(\mathbb{R}^{d}\right)$-measurable $f: \mathbb{R}^{d} \rightarrow \mathbb{R}$ which are bounded, or nonnegative are denoted by $\mathcal{B}_{b}\left(\mathbb{R}^{d}\right), \mathcal{B}^{+}\left(\mathbb{R}^{d}\right)$ respectively. Let $U \subset \mathbb{R}^{d}$, be an open set. The usual $L^{q}$-spaces $L^{q}(U, \mu), q \in$ $[1, \infty]$ of Borel measurable or classes of Borel measurable functions (depending on the context) are equipped with $L^{q}$-norm $\|\cdot\|_{L^{q}(U, \mu)}$ with respect to the measure $\mu$ on $U$ and $L_{l o c}^{q}\left(\mathbb{R}^{d}, \mu\right):=$ $\left\{f: f \cdot 1_{U} \in L^{q}\left(\mathbb{R}^{d}, \mu\right), \forall U \subset \mathbb{R}^{d}, U\right.$ relatively compact open $\}$, where $1_{A}$ denotes the indicator function of a set $A \subset \mathbb{R}^{d}$. Likewise $L_{l o c}^{q}\left(\mathbb{R}^{d}, \mathbb{R}^{d}, \mu\right)$ denotes the set of all locally $q$-fold integrable vector fields, i.e.

$$
L_{l o c}^{q}\left(\mathbb{R}^{d}, \mathbb{R}^{d}, \mu\right):=\left\{\mathbf{G}=\left(g_{1}, \ldots, g_{d}\right): \mathbb{R}^{d} \rightarrow \mathbb{R}^{d}: g_{i} \in L_{l o c}^{q}\left(\mathbb{R}^{d}, \mu\right), 1 \leq i \leq d\right\} .
$$


The Lebesgue measure on $\mathbb{R}^{d}$ is denoted by $d x$ and we write $L^{q}\left(\mathbb{R}^{d}\right), L_{l o c}^{q}\left(\mathbb{R}^{d}\right), L_{l o c}^{q}\left(\mathbb{R}^{d}, \mathbb{R}^{d}\right)$ for $L^{q}\left(\mathbb{R}^{d}, d x\right), L_{l o c}^{q}\left(\mathbb{R}^{d}, d x\right), L_{l o c}^{q}\left(\mathbb{R}^{d}, \mathbb{R}^{d}, d x\right)$ respectively. In order to avoid notational complications, we assume that locally integrable functions are whenever necessary pointwisely given (not for instance equivalence classes) and hence measurable. Moreover, whenever a function $f$ possesses a continuous version, we will assume it is given by it. However, if in a situation, it should be necessary or important to distinguish between classes and pointwisely given functions, we will mention it. If $\mathcal{A}$ is a set of measurable functions $f: \mathbb{R}^{d} \rightarrow \mathbb{R}$, we define $\mathcal{A}_{0}:=\{f \in \mathcal{A}$ : $\operatorname{supp}(f):=\operatorname{supp}(|f| d x)$ is compact in $\left.\mathbb{R}^{d}\right\}$ and $\mathcal{A}_{b}:=\mathcal{A} \cap L^{\infty}\left(\mathbb{R}^{d}\right)$. As usual, we also denote the set of continuous functions on $\mathbb{R}^{d}$, the set of continuous bounded functions on $\mathbb{R}^{d}$, the set of compactly supported continuous functions in $\mathbb{R}^{d}$ by $C\left(\mathbb{R}^{d}\right), C_{b}\left(\mathbb{R}^{d}\right), C_{0}\left(\mathbb{R}^{d}\right)$, respectively. Two Borel measurable functions $f$ and $g$ are called $\mu$-versions of each other, if $f=g \mu$-a.e.

Let $\nabla f:=\left(\partial_{1} f, \ldots, \partial_{d} f\right)$, where $\partial_{j} f$ is the $j$-th weak partial derivative of $f$ on $\mathbb{R}^{d}$ and $\partial_{i j} f:=$ $\partial_{i}\left(\partial_{j} f\right), i, j=1, \ldots, d$. The Sobolev space $H^{1, q}(U), q \in[1, \infty]$ is defined to be the set of all functions $f \in L^{q}(U)$ for which $\partial_{j} f \in L^{q}(U), j=1, \ldots, d$, and $H_{l o c}^{1, q}(U):=\{f: f \cdot \varphi \in$ $\left.H^{1, q}(U), \forall \varphi \in C_{0}^{\infty}(U)\right\}$. Here $C_{0}^{k}(U), k \in \mathbb{N} \cup\{\infty\}$, denotes the set of all $k$-fold continuously differentiable functions with compact support in $U$. Let $V$ be a bounded open set in $\mathbb{R}^{d}$ (typically a ball $B$ ) and $f: \bar{V} \rightarrow \mathbb{R}$ be a function. For $\beta \in(0,1)$ define

$$
\operatorname{höl}_{\beta}(f, \bar{V}):=\sup \left\{\frac{|f(x)-f(y)|}{\|x-y\|^{\beta}}: x, y \in \bar{V}, x \neq y\right\} \in[0, \infty],
$$

and the Hölder continuous functions of order $\beta \in(0,1)$ on $\bar{V}$ by

$$
C^{0, \beta}(\bar{V}):=\left\{f \in C(\bar{V}): \operatorname{höl}_{\beta}(f, \bar{V})<\infty\right\} .
$$

Then $C^{0, \beta}(\bar{V})$ is a Banach space with norm

$$
\|f\|_{C^{0, \beta}(\bar{V})}:=\sup _{x \in \bar{V}}|f(x)|+\operatorname{höl}_{\beta}(f, \bar{V}) .
$$

The space of all locally Hölder continuous functions of order $\beta \in(0,1)$ on $\mathbb{R}^{d}$ is defined by

$$
C_{l o c}^{0, \beta}\left(\mathbb{R}^{d}\right):=\left\{f: f \in C_{l o c}^{0, \beta}(\bar{B}) \text { for any ball } B\right\} .
$$

Let $Q$ be a bounded open set in $\mathbb{R}^{d} \times \mathbb{R}$ and $g: \bar{Q} \rightarrow \mathbb{R}$ be a function. For $\delta \in(0,1)$ denote

$$
\operatorname{phöl}_{\delta}(g, \bar{Q}):=\sup \left\{\frac{|g(x, t)-g(y, s)|}{(\|x-y\|+\sqrt{|t-s|})^{\delta}}:(x, t),(y, s) \in \bar{Q},(x, t) \neq(y, s)\right\} \in[0, \infty],
$$

and the parabolic Hölder continuous functions of order $\delta \in(0,1)$ on $\bar{Q}$ by

$$
C^{\delta ; \frac{\delta}{2}}(\bar{Q}):=\left\{g \in C(\bar{Q}): \operatorname{phöl}_{\delta}(g, \bar{Q})<\infty\right\} .
$$

Then $C^{\delta ; \frac{\delta}{2}}(\bar{Q})$ is a Banach space with norm

$$
\|g\|_{C^{\delta ; \frac{\delta}{2}}(\bar{Q})}:=\sup _{(x, t) \in \bar{Q}}|g(x, t)|+\operatorname{phöl}_{\delta}(g, \bar{Q}) .
$$

$g$ is called locally parabolic Hölder continuous, if for any bounded and open set $Q$, there exists $\delta=\delta(Q)$, such that $g \in C^{\delta ; \frac{\delta}{2}}(\bar{Q})$. Here $\delta$ may be different for different $Q$. In particular, if $t \in \mathbb{R}$ is fixed, we then say that $g(\cdot, t)$ is locally Hölder continuous with possibly changing Hölder exponents.

Given a function $f: \mathbb{R}^{d} \rightarrow \mathbb{R}$ and a Hunt process with cemetary $\Delta$, we follow the convention that $f$ is extended to $\mathbb{R}^{d} \cup\{\Delta\}$ by setting $f(\Delta)=0$. 


\section{Weak solutions via generalized Dirichlet forms and elliptic and parabolic regularity}

Let $\phi \in H_{l o c}^{1,2}\left(\mathbb{R}^{d}\right)$ be such that the measure $m:=\rho d x, \rho:=\phi^{2}$, has full support on $\mathbb{R}^{d}$. Let $H_{0}^{1,2}\left(\mathbb{R}^{d}, m\right)$ be the closure of $C_{0}^{\infty}\left(\mathbb{R}^{d}\right)$ in $L^{2}\left(\mathbb{R}^{d}, m\right)$ with respect to the norm $\left(\int_{\mathbb{R}^{d}}\left(\|\nabla f\|^{2}+\right.\right.$ $\left.\left.f^{2}\right) d m\right)^{1 / 2}$ and $H_{l o c}^{1,2}\left(\mathbb{R}^{d}, m\right):=\left\{f: f \cdot \varphi \in H_{0}^{1,2}\left(\mathbb{R}^{d}, m\right), \forall \varphi \in C_{0}^{\infty}\left(\mathbb{R}^{d}\right)\right\}$. Let $A=\left(a_{i j}\right)_{1 \leq i, j \leq d}$ with $a_{i j} \in H_{l o c}^{1,2}\left(\mathbb{R}^{d}, m\right)$ be a symmetric matrix of functions and locally uniformly strictly elliptic, i.e. for every (open) ball $B \subset \mathbb{R}^{d}$ there exist real numbers $\lambda_{B}, \Lambda_{B}>0$, such that

$$
\lambda_{B}\|\xi\|^{2} \leq\langle A(x) \xi, \xi\rangle \leq \Lambda_{B}\|\xi\|^{2} \text { for all } \xi \in \mathbb{R}^{d}, x \in B .
$$

Let $\mathbf{G}=\left(g_{1}, \ldots, g_{d}\right) \in L_{l o c}^{2}\left(\mathbb{R}^{d}, \mathbb{R}^{d}, m\right)$ be such that with

$$
L f:=\frac{1}{2} \sum_{i, j=1}^{d} a_{i j} \partial_{i} \partial_{j} f+\sum_{i=1}^{d} g_{i} \partial_{i} f, f \in C_{0}^{\infty}\left(\mathbb{R}^{d}\right),
$$

it holds

$$
\int_{\mathbb{R}^{d}} L f d m=0, \quad \forall f \in C_{0}^{\infty}\left(\mathbb{R}^{d}\right)
$$

Then it is shown in [30, Theorem 1.5] that there exists a closed extension $\left(L_{1}, D\left(L_{1}\right)\right)$ on $L^{1}\left(\mathbb{R}^{d}, m\right)$ of $\left(L, C_{0}^{\infty}\left(\mathbb{R}^{d}\right)\right)$ that generates a sub-Markovian $C_{0}$-semigroup of contractions $\left(T_{t}\right)_{t>0}$. Restricting $\left(T_{t}\right)_{t>0}$ to $L^{1}\left(\mathbb{R}^{d}, m\right)_{b}$, it is well-known that $\left(T_{t}\right)_{t>0}$ can be extended to a subMarkovian $C_{0}$-semigroup of contractions on each $L^{r}\left(\mathbb{R}^{d}, m\right), r \in[1, \infty)$. Denote by $\left(L_{r}, D\left(L_{r}\right)\right)$ the corresponding closed generator with graph norm

$$
\|f\|_{D\left(L_{r}\right)}:=\|f\|_{L^{r}\left(\mathbb{R}^{d}, m\right)}+\left\|L_{r} f\right\|_{L^{r}\left(\mathbb{R}^{d}, m\right)},
$$

and by $\left(G_{\alpha}\right)_{\alpha>0}$ the corresponding resolvent. For $\left(T_{t}\right)_{t>0}$ and $\left(G_{\alpha}\right)_{\alpha>0}$ we do not explicitly denote in the notation on which $L^{r}\left(\mathbb{R}^{d}, m\right)$-space they act. We assume that this is clear from the context. Moreover, $\left(T_{t}\right)_{t>0}$ and $\left(G_{\alpha}\right)_{\alpha>0}$ can be uniquely defined on $L^{\infty}\left(\mathbb{R}^{d}, m\right)$, but are no longer strongly continuous there.

Writing

$$
L f=\frac{1}{2} \sum_{i, j=1}^{d} a_{i j} \partial_{i} \partial_{j} f+\sum_{i=1}^{d} \beta_{i}^{\rho, A} \partial_{i} f+\sum_{i=1}^{d}\left(g_{i}-\beta_{i}^{\rho, A}\right) \partial_{i} f
$$

with

$$
\beta_{i}^{\rho, A}:=\frac{1}{2} \sum_{j=1}^{d}\left(\partial_{j} a_{i j}+a_{i j} \frac{\partial_{j} \rho}{\rho}\right), 1 \leq i \leq d, \beta^{\rho, A}:=\left(\beta_{1}^{\rho, A}, \ldots, \beta_{d}^{\rho, A}\right)
$$

we observe that (6) is equivalent to

$$
\int_{\mathbb{R}^{d}}\left\langle\mathbf{G}-\beta^{\rho, A}, \nabla f\right\rangle d m=0, \quad \forall f \in C_{0}^{\infty}\left(\mathbb{R}^{d}\right),
$$


hence

$$
\int_{\mathbb{R}^{d}} \widehat{L} f d m=0, \quad \forall f \in C_{0}^{\infty}\left(\mathbb{R}^{d}\right)
$$

where

$$
\widehat{L} f=\frac{1}{2} \sum_{i, j=1}^{d} a_{i j} \partial_{i} \partial_{j} f+\sum_{i=1}^{d} \beta_{i}^{\rho, A} \partial_{i} f-\sum_{i=1}^{d}\left(g_{i}-\beta_{i}^{\rho, A}\right) \partial_{i} f .
$$

Noting that $\widehat{g}_{i}:=2 \beta_{i}^{\rho, A}-g_{i} \in L_{l o c}^{2}\left(\mathbb{R}^{d}, m\right)$, we see that $L$ and $\widehat{L}$ have the same structural properties, i.e. they are given as the sum of a symmetric second order elliptic differential operator and a divergence free first order perturbation with same integrability condition with respect to the measure $m$. Therefore all what will be derived below for $L$ will hold analogously for $\widehat{L}$. Denote the operators corresponding to $\widehat{L}$ (again defined through [30, Theorem 1.5]) by $\left(\widehat{L}_{r}, D\left(\widehat{L}_{r}\right)\right)$ for the co-generator on $L^{r}\left(\mathbb{R}^{d}, m\right), r \in[1, \infty),\left(\widehat{T}_{t}\right)_{t>0}$ for the co-semigroup, $\left(\widehat{G}_{\alpha}\right)_{\alpha>0}$ for the coresolvent. By [30, Section 3], we obtain a corresponding bilinear form with domain $D\left(L_{2}\right) \times$ $L^{2}\left(\mathbb{R}^{d}, m\right) \cup L^{2}\left(\mathbb{R}^{d}, m\right) \times D\left(\widehat{L}_{2}\right)$ by

$$
\mathcal{E}(f, g):= \begin{cases}-\int_{\mathbb{R}^{d}} L_{2} f \cdot g d m & \text { for } f \in D\left(L_{2}\right), g \in L^{2}\left(\mathbb{R}^{d}, m\right), \\ -\int_{\mathbb{R}^{d}} f \cdot \widehat{L}_{2} g d m & \text { for } f \in L^{2}\left(\mathbb{R}^{d}, m\right), g \in D\left(\widehat{L}_{2}\right) .\end{cases}
$$

$\mathcal{E}$ is called the generalized Dirichlet form associated with $\left(L_{2}, D\left(L_{2}\right)\right)$. Using integration by parts, it is easy to see that

$$
\mathcal{E}(f, g)=\frac{1}{2} \int_{\mathbb{R}^{d}}\langle A \nabla f, \nabla g\rangle d m-\int_{\mathbb{R}^{d}}\left\langle\mathbf{G}-\beta^{\rho, A}, \nabla f\right\rangle g d m, \quad f, g \in C_{0}^{\infty}\left(\mathbb{R}^{d}\right) .
$$

The following lemma, see [30, Remark 1.7(iii)], will be used later:

Lemma 3.1 Let $u \in D\left(L_{1}\right)_{b}$. Then $u^{2} \in D\left(L_{1}\right)_{b}$ and

$$
L_{1} u^{2}=\langle A \nabla u, \nabla u\rangle+2 u L_{1} u .
$$

We are going to restrict our previous assumptions to the ones of the following theorem. The theorem itself is an immediate consequence of an important result [7, Theorem 2.4.1] (see also [8, Theorem 1] for the original result), which itself is derived by using elliptic regularity results from [32] in an essential way.

Theorem 3.2 Let $p>d$ be arbitrary but fixed. Let $A:=\left(a_{i j}\right)_{1 \leq i, j \leq d}$ be a symmetric $d \times d$ matrix of functions $a_{i j} \in H_{l o c}^{1, p}\left(\mathbb{R}^{d}\right)$ satisfying (4). Let $\mathbf{G}=\left(g_{1}, \ldots, g_{d}\right) \in L_{l o c}^{p}\left(\mathbb{R}^{d}, \mathbb{R}^{d}\right)$. Then there exists $\rho \in C_{\text {loc }}^{0,1-d / p}\left(\mathbb{R}^{d}\right) \cap H_{\text {loc }}^{1, p}\left(\mathbb{R}^{d}\right)$ with $\rho(x)>0$ for all $x \in \mathbb{R}^{d}$ and such that

$$
\int_{\mathbb{R}^{d}}\left\langle\mathbf{G}-\beta^{\rho, A}, \nabla \varphi\right\rangle \rho d x=0, \quad \forall \varphi \in C_{0}^{\infty}\left(\mathbb{R}^{d}\right),
$$

with

$$
\beta^{\rho, A} \in L_{l o c}^{p}\left(\mathbb{R}^{d}, \mathbb{R}^{d}\right)
$$

In particular, setting

$$
\mathbf{B}=\left(b_{1}, \ldots, b_{d}\right):=\mathbf{G}-\beta^{\rho, A},
$$

we have obtained a representation of an arbitrary $\mathbf{G} \in L_{l o c}^{p}\left(\mathbb{R}^{d}, \mathbb{R}^{d}\right)$ as the sum of the logarithmic derivative $\beta^{\rho, A}$ associated to $A$ and $\rho$ and a $\rho d x$-divergence free vector field $\mathbf{B} \in L_{l o c}^{p}\left(\mathbb{R}^{d}, \mathbb{R}^{d}\right)$, namely

$$
\mathbf{G}=\beta^{\rho, A}+\mathbf{B}
$$


Remark 3.3 It is possible and not difficult to generalize Theorem 3.2 (and basically everything that follows below) in two directions. We do not do this here because it only leads to technical and notational complications, which are better to be investigated and overcome elsewhere. But all necessary tools can be found in this work. The two directions are:

(i) Theorem 3.2 also holds with $\mathbb{R}^{d}$ replaced by any open set $U \subset \mathbb{R}^{d}, H_{\text {loc }}^{1, p}(U)$ defined as in Section 2, and

$$
\begin{aligned}
L_{l o c}^{p}(U) & :=\left\{f: f 1_{V} \in L^{p}(U), \forall V \text { relatively compact open with } \bar{V} \subset U\right\}, \\
C_{l o c}^{0,1-d / p}(U) & :=\left\{f: f \in C^{0,1-d / p}(\bar{V}), \forall \text { V relatively compact open with } \bar{V} \subset U\right\},
\end{aligned}
$$

by considering an exhaustion with bounded and open sets $\left(V_{n}\right)_{n \geq 1}$ of $U$, i.e.

$$
V_{n} \subset \bar{V}_{n} \subset V_{n+1} \text { for all } n \in \mathbb{N} \text { and } \cup_{n=1}^{\infty} V_{n}=U \text {. }
$$

(ii) As in [7, Theorem 2.4.1], the regularity conditions on $a_{i j}, g_{i}, 1 \leq i, j \leq d$, can be generalized to $a_{i j} \in H^{1, p_{n}}\left(B_{n}\right)$ and $g_{i} \in L^{p_{n}}\left(B_{n}\right)$ with $p_{n}>d$. The only interesting case is when $\lim _{n \rightarrow \infty} p_{n}=d$, which leads to a slight but technical improvement of the conditions of Theorem 3.2. Note that $\left(B_{n}\right)_{n \geq 1}$ here is a special exhaustion with bounded and open sets of $\mathbb{R}^{d}$ but one can generalize this to an arbitrary exhaustion with bounded and open sets $\left(V_{n}\right)_{n \geq 1}$ of $\mathbb{R}^{d}$.

From now on unless otherwise stated, we fix one density $\rho$ as in Theorem 3.2 and hence assume that

$$
A:=\left(a_{i j}\right)_{1 \leq i, j \leq d}, \mathbf{G}=\left(g_{1}, \ldots, g_{d}\right), \beta^{\rho, A}=\left(\beta_{1}^{\rho, A}, \ldots, \beta_{d}^{\rho, A}\right), \mathbf{B}=\left(b_{1}, \ldots, b_{d}\right),
$$

are as in Theorem 3.2 with

$$
p>d .
$$

This implies all assumptions prior to Theorem 3.2 and we fix from now on the corresponding generalized Dirichlet form $\mathcal{E}$ associated with $\left(L_{2}, D\left(L_{2}\right)\right)$ and all the corresponding objects under the assumptions of Theorem 3.2 . As before, we set

$$
m:=\rho d x
$$

Note, that due to the properties of $\rho$ in Theorem 3.2, we have that $L_{l o c}^{p}\left(\mathbb{R}^{d}\right)=L_{l o c}^{p}\left(\mathbb{R}^{d}, m\right)$ as well as $L_{l o c}^{p}\left(\mathbb{R}^{d}, \mathbb{R}^{d}\right)=L_{l o c}^{p}\left(\mathbb{R}^{d}, \mathbb{R}^{d}, m\right)$.

We will use the following result from [5, Theorem 5.1], adapted to our needs.

Proposition 3.4 Let $d \geq 2$ and $\mu$ a locally finite (signed) Borel measure on $\mathbb{R}^{d}$ that is absolutely continuous with respect to Lebesgue measure on $\mathbb{R}^{d}$. Let $A=\left(a_{i j}\right)_{1 \leq i, j \leq d}$ and $p>d$ be as in Theorem 3.2. Let $h_{i}, c, f \in L_{l o c}^{p}\left(\mathbb{R}^{d}\right)$ and assume that

$$
\int_{\mathbb{R}^{d}}\left(\sum_{i, j=1}^{d} \frac{a_{i j}}{2} \partial_{i j} \varphi+\sum_{i=1}^{d} h_{i} \partial_{i} \varphi+c \varphi\right) d \mu=\int_{\mathbb{R}^{d}} \varphi f d x, \quad \forall \varphi \in C_{0}^{\infty}\left(\mathbb{R}^{d}\right),
$$

where $h_{i}, c$ are locally $\mu$-integrable. Then $\mu$ has a density in $H_{l o c}^{1, p}\left(\mathbb{R}^{d}\right)$ that is locally Hölder continuous. 
We further state a result originally due to Morrey (see the wrong statement in the original monograph [23, Theorem 5.5.5'] and [7, Theorem 1.7.4] and Corollaries for its correction).

Proposition 3.5 Assume $p>d \geq 2$. Let $B^{\prime} \subset \mathbb{R}^{d}$ be a ball, $h=\left(h_{1}, \ldots, h_{d}\right): B^{\prime} \rightarrow \mathbb{R}^{d}$ and $c, e: B^{\prime} \rightarrow \mathbb{R}$ such that

$$
h_{i} \in L^{p}\left(B^{\prime}\right), 1 \leq i \leq d, \quad \text { and } c, e \in L^{q}\left(B^{\prime}\right) \text { for } q:=\frac{d p}{d+p} .
$$

Let $A=\left(a_{i j}\right)_{1 \leq i, j \leq d}$ be as in Theorem 3.2. Assume that $u \in H^{1, p}\left(B^{\prime}\right)$ is a solution of

$$
\int_{B^{\prime}} \sum_{i=1}^{d}\left(\partial_{i} \varphi\left(\sum_{j=1}^{d} \frac{a_{i j}}{2} \partial_{j} u+h_{i} u\right)\right)+\varphi(c u+e) d x=0, \quad \forall \varphi \in C_{0}^{\infty}\left(B^{\prime}\right),
$$

Then for every ball $B$ with $\bar{B} \subset B^{\prime}$, we obtain the estimate

$$
\|u\|_{H^{1, p}(B)} \leq c_{0}\left(\|e\|_{L^{q}\left(B^{\prime}\right)}+\|u\|_{L^{1}\left(B^{\prime}\right)}\right)
$$

where $c_{0}<\infty$ is some constant independent of $e$ and $u$.

Now, we will apply the standard arguments from [1] whose details have been exposed in a very clear way in [5]. We will briefly explain (until and including Remark 3.7) the line of arguments how Propositions 3.4 and 3.5 lead to elliptic regularity results for $\left(G_{\alpha}\right)_{\alpha>0}$ and $\left(T_{t}\right)_{t>0}$ by using well-known arguments (see for instance [1], [5], or [27]). However, as we will see later, we will slightly improve some regularity results compared to the just mentioned papers. First, we choose an arbitrary $g \in C_{0}^{\infty}\left(\mathbb{R}^{d}\right), \alpha>0$. Applying Proposition 3.4 with

$$
\mu=-\rho G_{\alpha} g d x, h_{i}=\beta_{i}^{\rho, A}-b_{i}, 1 \leq i \leq d, c=-\alpha, f=g \rho \in L_{l o c}^{p}\left(\mathbb{R}^{d}\right),
$$

we obtain $\rho G_{\alpha} g \in H_{l o c}^{1, p}\left(\mathbb{R}^{d}\right)$. Then, we apply Proposition 3.5 with

$$
u=\rho G_{\alpha} g, h_{i}=\sum_{j=1}^{d}\left(\frac{\partial_{j} a_{i j}}{2}-\left(\beta_{i}^{\rho, A}-b_{i}\right)\right), 1 \leq i \leq d,
$$

and

$$
c=\alpha, e=\rho g \in L^{q}\left(B^{\prime}\right)
$$

where

$$
q:=\frac{d p}{d+p} \in(d / 2, p / 2)
$$

By the properties of $\rho$, we obtain

$$
\left\|\rho G_{\alpha} g\right\|_{H^{1, p}(B)} \leq c_{0}\left(\|g\|_{L^{q}\left(B^{\prime}, m\right)}+\left\|G_{\alpha} g\right\|_{L^{1}\left(B^{\prime}, m\right)}\right),
$$

where $c_{0}$ is possibly different form the constant in Proposition 3.5, but also doesn't depend on $g$. The last inequality is easily seen to extend to $g \in L^{r}\left(\mathbb{R}^{d}, m\right), r \in[q, \infty]$, using the contraction properties of $\left(G_{\alpha}\right)_{\alpha>0}$. From that we then get that for any $r \in[q, \infty], \alpha>0$

$$
\left\|\rho G_{\alpha} g\right\|_{H^{1, p}(B)} \leq c_{0}\left(\|g\|_{L^{r}\left(B^{\prime}, m\right)}+\left\|G_{\alpha} g\right\|_{L^{1}\left(B^{\prime}, m\right)}\right), \quad \forall g \in L^{r}\left(\mathbb{R}^{d}, m\right),
$$


where $c_{0}$ is a constant that may be different for different $\alpha$ and $r$, but doesn't depend on $g$. Using the contraction properties of $\left(G_{\alpha}\right)_{\alpha>0}$, (13) immediately implies

$$
\left\|\rho G_{\alpha} g\right\|_{H^{1, p}(B)} \leq c_{0}\|g\|_{L^{r}\left(\mathbb{R}^{d}, m\right)}, \quad \forall g \in L^{r}\left(\mathbb{R}^{d}, m\right),
$$

where $c_{0}$ in (13) may be different from $c_{0}$ in (14) but has the same properties.

Writing $T_{0}:=i d$ and

$$
T_{t} f=G_{1}\left(1-L_{r}\right) T_{t} f, f \in D\left(L_{r}\right), r \in[q, \infty), t \geq 0,
$$

we can see by (13) that for any $r \in[q, \infty), t \geq 0$

$$
\left\|\rho T_{t} f\right\|_{H^{1, p}(B)} \leq c_{0}\left\|T_{t} f\right\|_{D\left(L_{r}\right)}, \quad \forall f \in D\left(L_{r}\right),
$$

where $c_{0}$ is a constant that may be different for different $r$, but doesn't depend on $f$.

By Morrey's inequality applied to an arbitrary ball $B$, there exists a constant $c>0$ independent of $f$ such that

$$
\|\widetilde{f}\|_{C^{0, \beta}(\bar{B})} \leq c\|f\|_{H^{1, p}(B)}, \quad \forall f \in H^{1, p}(B),
$$

where $\tilde{f}$ on the left hand side is the unique continuous $d x$-version of $f \in H^{1, p}(B)$ and

$$
\beta:=1-d / p .
$$

In our situation $\rho \in C^{0, \beta}(\bar{B})$ for any ball $B \subset \mathbb{R}^{d}$ and since $\inf _{x \in \bar{B}} \rho(x)>0$, we obtain that $\frac{1}{\rho} \in C^{0, \beta}(\bar{B})$. Now for $f, g \in C^{0, \beta}(\bar{B})$ it holds $f \cdot g \in C^{0, \beta}(\bar{B})$ and

$$
\|f \cdot g\|_{C^{0, \beta}(\bar{B})} \leq\|f\|_{C^{0, \beta}(\bar{B})}\|g\|_{C^{0, \beta}(\bar{B})} .
$$

For any ball $B, t \geq 0, \alpha>0, g \in L^{r}\left(\mathbb{R}^{d}, m\right), r \in[q, \infty], f \in D\left(L_{r}\right), r \in[q, \infty)$

$$
\left\|\rho G_{\alpha} g\right\|_{H^{1, p}(B)},\left\|\rho T_{t} f\right\|_{H^{1, p}(B)}
$$

are bounded and so by Morrey's inequality applied to each ball $B$ and (17) there exist unique locally Hölder continuous $m$-versions $R_{\alpha} g, P_{t} f$ of $G_{\alpha} g, T_{t} f$, where we set

$$
P_{0}:=i d,
$$

with

$$
\left\|R_{\alpha} g\right\|_{C^{0, \beta}(\bar{B})} \leq\left\|\rho^{-1}\right\|_{C^{0, \beta}(\bar{B})}\left\|\rho R_{\alpha} g\right\|_{C^{0, \beta}(\bar{B})} \leq\left\|\rho^{-1}\right\|_{C^{0, \beta}(\bar{B})} c\left\|\rho G_{\alpha} g\right\|_{H^{1, p}(B)}
$$

and

$$
\left\|P_{t} f\right\|_{C^{0, \beta}(\bar{B})} \leq\left\|\rho^{-1}\right\|_{C^{0, \beta}(\bar{B})} c\left\|\rho T_{t} f\right\|_{H^{1, p}(B)} .
$$

Applying (13), (14), (15) to the last two inequalities, we get for any $t \geq 0, \alpha>0, g \in L^{r}\left(\mathbb{R}^{d}, m\right)$, $r \in[q, \infty], f \in D\left(L_{r}\right), r \in[q, \infty)$, and any ball $B^{\prime}$ with $\bar{B} \subset B^{\prime}$

$$
\begin{gathered}
\left\|R_{\alpha} g\right\|_{C^{0, \beta}(\bar{B})} \leq c_{0}\left(\|g\|_{L^{r}\left(B^{\prime}, m\right)}+\left\|G_{\alpha} g\right\|_{L^{1}\left(B^{\prime}, m\right)}\right), \\
\left\|R_{\alpha} g\right\|_{C^{0, \beta}(\bar{B})} \leq c_{0}\|g\|_{L^{r}\left(\mathbb{R}^{d}, m\right)}, \\
\left\|P_{t} f\right\|_{C^{0, \beta}(\bar{B})} \leq c_{0}\left\|T_{t} f\right\|_{D\left(L_{r}\right)},
\end{gathered}
$$

where $c_{0}$ is a constant that may be different for different $r$ (and different in each inequality (18), (19), and (201) ), but doesn't depend on $f$, nor on $g$. We summarize consequences of the derived estimates in the following proposition. 
Proposition 3.6 Let $t \geq 0, \alpha>0$ be arbitrary and $q, \beta$ be defined as in (12), (16). Then under the conditions of Theorem 3.2, it holds:

(i) $G_{\alpha} g$ has a locally Hölder continuous m-version

$$
R_{\alpha} g \in C_{l o c}^{0, \beta}\left(\mathbb{R}^{d}\right), \quad \forall g \in \bigcup_{r \in[q, \infty]} L^{r}\left(\mathbb{R}^{d}, m\right)
$$

(ii) $T_{t} f$ has a locally Hölder continuous m-version

$$
P_{t} f \in C_{l o c}^{0, \beta}\left(\mathbb{R}^{d}\right), \quad \forall f \in \bigcup_{r \in[q, \infty)} D\left(L_{r}\right) .
$$

(iii) For any $f \in \bigcup_{r \in[q, \infty)} D\left(L_{r}\right)$ the map

$$
(x, t) \mapsto P_{t} f(x)
$$

is continuous on $\mathbb{R}^{d} \times[0, \infty)$.

Proof (i) and (ii) are direct consequences of (18), (19), (20). In order to show (iii), let $f \in D\left(L_{r}\right)$ for some $r \geq q$ and $\left(\left(x_{n}, t_{n}\right)\right)_{n \geq 1}$ be a sequence in $\mathbb{R}^{d} \times[0, \infty)$ that converges to $\left(x_{0}, t_{0}\right) \in$ $\mathbb{R}^{d} \times[0, \infty)$. Then there exists a ball $B$ such that $x_{n} \in \bar{B}$ for all $n \geq 0$. By (20) applied with $t=0$ to $P_{t_{n}} f-P_{t_{0}} f \in D\left(L_{r}\right)$, noting that $L_{r}\left(P_{t_{n}} f-P_{t_{0}} f\right)=P_{t_{n}} L_{r} f-P_{t_{0}} L_{r} f$ and using the continuity for each $g \in L^{r}\left(\mathbb{R}^{d}, m\right)$ of $t \mapsto P_{t} g$ on $[0, \infty)$, we obtain that $P_{t_{n}} f \rightarrow P_{t_{0}} f$ in $C^{0, \beta}(\bar{B})$. Then it is clear from (ii) that

$$
\left|P_{t_{n}} f\left(x_{n}\right)-P_{t_{0}} f\left(x_{0}\right)\right| \leq\left|P_{t_{n}} f\left(x_{n}\right)-P_{t_{0}} f\left(x_{n}\right)\right|+\left|P_{t_{0}} f\left(x_{n}\right)-P_{t_{0}} f\left(x_{0}\right)\right|
$$

converges to zero as $n \rightarrow \infty$.

Remark 3.7 $\quad$ (i) In comparison to [1], [5], 27], we obtained in Proposition [3.6] (i) that $\left(G_{\alpha}\right)_{\alpha>0}$ is $L^{r}\left(\mathbb{R}^{d}, m\right)$-strong Feller for any $r \in[q, \infty]$, which is an improvement to the mentioned papers since there it is only obtained for $r \in[p, \infty]$. This plays a role, since it will imply (2) for $r=2$. Indeed, we will see later in Lemma 3.15(ii) that $\int_{0}^{t}|f|^{2}\left(X_{s}\right)$ ds is finite in the sense of (2), whenever $f \in L_{\text {loc }}^{2 q}\left(\mathbb{R}^{d}\right)$. But $2 q \in(d, p)$, hence $L_{l o c}^{p}\left(\mathbb{R}^{d}\right) \subset L_{\text {loc }}^{2 q}\left(\mathbb{R}^{d}\right)$.

(ii) We can use Proposition 3.6(i) to get a resolvent kernel and a resolvent kernel density for any $x \in \mathbb{R}^{d}$. Indeed, for any $\alpha>0, x \in \mathbb{R}^{d}$, Proposition [3.6(i) implies that

$$
R_{\alpha}(x, A):=\lim _{l \rightarrow \infty} R_{\alpha}\left(1_{B_{l} \cap A}\right)(x), A \in \mathcal{B}\left(\mathbb{R}^{d}\right)
$$

defines a finite measure $R_{\alpha}(x, d y)$ on $\left(\mathbb{R}^{d}, \mathcal{B}\left(\mathbb{R}^{d}\right)\right.$ ) (such that $\alpha R_{\alpha}(x, d y)$ is a sub-probability measure) that is absolutely continuous with respect to $m$. The Radon-Nikodym derivative

$$
r_{\alpha}(x, \cdot):=\frac{R_{\alpha}(x, d y)}{m(d y)}
$$

then defines the desired resolvent kernel density. 
(iii) If the $L^{2}\left(\mathbb{R}^{d}, m\right)$-semigroup $\left(T_{t}\right)_{t>0}$ is analytic (for instance, if the bilinear form in (11) satisfies a sector condition) then by Stein interpolation $\left(T_{t}\right)_{t>0}$ is also analytic on $L^{r}\left(\mathbb{R}^{d}, m\right)$ for any $r \in(2, \infty)$ (cf. [27, Remark 2.5]). Hence by [24, Ch. 2, Theorem 5.2(d)], we have for any $r \in[2, \infty), f \in L^{r}\left(\mathbb{R}^{d}, m\right)$

$$
T_{t} f \in D\left(L_{r}\right), \quad \text { and } \quad\left\|L_{r} T_{t} f\right\|_{L^{r}\left(\mathbb{R}^{d}, m\right)} \leq \frac{\text { const. }}{t}\|f\|_{L^{r}\left(\mathbb{R}^{d}, m\right)} .
$$

Therefore, (20) can be improved and extended as follows: for any $r \in[q \vee 2, \infty), t>0$, $f \in L^{r}\left(\mathbb{R}^{d}, m\right)$ and any ball $B$

$$
\left\|P_{t} f\right\|_{C^{0, \beta}(\bar{B})} \leq c_{0}\left(1+\frac{\text { const. }}{t}\right)\|f\|_{L^{r}\left(\mathbb{R}^{d}, m\right)} .
$$

We can then use (23) to get a heat kernel and a heat kernel density for any $x \in \mathbb{R}^{d}$. Indeed, for any $t>0, x \in \mathbb{R}^{d}$, (23) implies that

$$
P_{t}(x, A):=\lim _{l \rightarrow \infty} P_{t}\left(1_{B_{l} \cap A}\right)(x), A \in \mathcal{B}\left(\mathbb{R}^{d}\right)
$$

defines a sub-probability measure $P_{t}(x, d y)$ on $\left(\mathbb{R}^{d}, \mathcal{B}\left(\mathbb{R}^{d}\right)\right)$ that is absolutely continuous with respect to $m$. The Radon-Nikodym derivative

$$
p_{t}(x, \cdot):=\frac{P_{t}(x, d y)}{m(d y)}
$$

then defines the desired heat kernel density. However, in general $\left(T_{t}\right)_{t>0}$ is not analytic and therefore we cannot impose analyticity. Moreover, it is in general difficult to check analyticity, in particular the sector condition of the corresponding bilinear form (see for instance [27, Section 5]).

Unfortunately, by what is explained in Remark 3.7(iii) the semigroup estimate (20) which leads to Proposition 3.6(ii) seems just not good enough to obtain a pointwise heat kernel from which one could then try to build a transition function of a nice Markov process. We will proceed by deriving more regularity in the following Theorem 3.8.

Theorem 3.8 Let $A:=\left(a_{i j}\right)_{1 \leq i, j \leq d}, \mathbf{G}, \rho, \beta^{\rho, A}$, and $\mathbf{B}$ be as in Theorem 3.2. For each $s \in$ $[1, \infty]$, consider the $L^{s}\left(\mathbb{R}^{d}, m\right)$-semigroup $\left(T_{t}\right)_{t>0}$. Then for any $f \in L^{s}\left(\mathbb{R}^{d}, m\right)$ and $t>0$, $T_{t} f$ has a continuous m-version $P_{t} f$ on $\mathbb{R}^{d}$. More precisely, P.f(.) is locally parabolic Hölder continuous on $\mathbb{R}^{d} \times(0, \infty)$ and for any bounded open sets $U, V$ in $\mathbb{R}^{d}$ with $\bar{U} \subset V$ and $0<\tau_{3}<$ $\tau_{1}<\tau_{2}<\tau_{4}$, i.e. $\left[\tau_{1}, \tau_{2}\right] \subset\left(\tau_{3}, \tau_{4}\right)$, we have for some $\gamma \in(0,1)$ the following estimate for all $f \in \cup_{s \in[1, \infty]} L^{s}\left(\mathbb{R}^{d}, m\right)$ with $f \geq 0$,

$$
\|P . f(\cdot)\|_{C^{\gamma ; \frac{\gamma}{2}}\left(\bar{U} \times\left[\tau_{1}, \tau_{2}\right]\right)} \leq C_{6}\|P . f(\cdot)\|_{L^{1}\left(V \times\left(\tau_{3}, \tau_{4}\right), m \otimes d t\right)},
$$

where $C_{6}, \gamma$ are constants that depend on $\bar{U} \times\left[\tau_{1}, \tau_{2}\right], V \times\left(\tau_{3}, \tau_{4}\right)$, but are independent of $f$.

Proof First assume $f \in C_{0}^{\infty}\left(\mathbb{R}^{d}\right), f \geq 0$ and set $u(x, t):=\rho(x) P_{t} f(x)$. Then $f \in D\left(L_{p}\right)$ and by Proposition 3.6(iii) $P_{t} f(x)$ is jointly continuous on $\mathbb{R}^{d} \times[0, \infty)$. Therefore the same is true for $u(x, t)$. Let $\widehat{L}$ be as in (10) and $T>0$ be arbitrary. Then exactly as in [6, (4.7)] (note that there 
the underlying measure $m=\mu$ is a probability measure but it doesn't matter), we get for any $\varphi \in C_{0}^{\infty}\left(\mathbb{R}^{d} \times(0, T)\right)$

$$
0=-\int_{0}^{T} \int_{\mathbb{R}^{d}}\left(\partial_{t} \varphi+\widehat{L} \varphi\right) u d x d t
$$

Note that $u \in H^{1,2}(O \times(0, T))$ for any bounded and open set $O \subset \mathbb{R}^{d}$. We can hence use integration by parts in the right hand term of (27) and see that

$$
0=\int_{0}^{T} \int_{\mathbb{R}^{d}}\left(\frac{1}{2}\langle A \nabla u, \nabla \varphi\rangle+u\langle\beta, \nabla \varphi\rangle-u \partial_{t} \varphi\right) d x d t
$$

where $\beta:=\frac{1}{2} \nabla A+\mathbf{G}-2 \beta^{\rho, A} \in L_{l o c}^{p}\left(\mathbb{R}^{d}, \mathbb{R}^{d}\right),(\nabla A)_{i}:=\sum_{j=1}^{d} \partial_{j} a_{i j}, 1 \leq i \leq d$.

Let $\tau_{2}^{*}:=\frac{\tau_{2}+\tau_{4}}{2}$ and take $r>0$ so that

$$
r<\min \left(\frac{1}{9} \sqrt{\frac{\tau_{4}-\tau_{2}}{14}}, \frac{1}{9} \sqrt{\frac{\tau_{1}}{2}}\right) \quad \text { and } \quad R_{\bar{x}}(9 r) \subset V, \forall \bar{x} \in \bar{U} .
$$

Then for all $(\bar{x}, \bar{t}) \in \bar{U} \times\left[\tau_{1}, \tau_{2}^{*}\right]$, we have $\bar{t}-2(9 r)^{2}>0$ and

$$
\left.R_{\bar{x}}(9 r) \times\left(\bar{t}+6(9 r)^{2}, \bar{t}+7(9 r)^{2}\right)\right) \subset V \times\left(\tau_{3}, \tau_{4}\right) .
$$

Using [2, Theorem 4], for any $(x, t),(y, s) \in R_{\bar{x}}(r) \times\left(\bar{t}-r^{2}, \bar{t}\right)$ we have

$$
|u(x, t)-u(y, s)| \leq C_{1} r^{-\gamma}(\|x-y\|+\sqrt{|t-s|})^{\gamma} \sup _{R_{\bar{x}}(3 r) \times\left(\bar{t}-(3 r)^{2}, \bar{t}\right)} u
$$

where $C_{1}$ and $\gamma \leq 1-\frac{d}{p}$ are constants independent of $f, r$ and $(\bar{x}, \bar{t})$. Thus $u \in C^{\gamma ; \frac{\gamma}{2}}\left(\bar{R}_{r}(\bar{x}) \times\right.$ $\left.\left[\bar{t}-r^{2}, \bar{t}\right]\right)$ and

$$
\|u\|_{C^{\gamma ; \frac{\gamma}{2}}\left(\bar{R}_{r}(\bar{x}) \times\left[\bar{t}-r^{2}, \bar{t}\right]\right)} \leq\left(1+C_{1} r^{-\gamma}\right) \sup _{R_{\bar{x}}(3 r) \times\left(\bar{t}-(3 r)^{2}, \bar{t}\right)} u .
$$

Using the compactness of $\bar{U} \times\left[\tau_{1}, \tau_{2}\right]$, there exist $\left(x_{i}, t_{i}\right) \in \bar{U} \times\left[\tau_{1}, \tau_{2}^{*}\right], i=1, \ldots, N$, such that

$$
\bar{U} \times\left[\tau_{1}, \tau_{2}\right] \subset \bigcup_{i=1}^{N} R_{x_{i}}(r) \times\left(t_{i}-r^{2}, t_{i}\right)=: Q .
$$

Take a smooth partition of unity $\left(\phi_{i}\right)_{i=1, \ldots, N}$ subordinate to $\left(R_{x_{i}}(r) \times\left(t_{i}-r^{2}, t_{i}\right)\right)_{i=1, \ldots, N}$. For each $1 \leq i \leq N, \phi_{i} u \in C^{\gamma ; \frac{\gamma}{2}}(\bar{Q})$, so that $u=\sum_{i=1}^{N} \phi_{i} u$ in $\bar{U} \times\left[\tau_{1}, \tau_{2}\right]$ implies $u \in C^{\gamma ; \frac{\gamma}{2}}(\bar{U} \times$ $\left.\left[\tau_{1}, \tau_{2}\right]\right)$. Furthermore, we have

$$
\begin{aligned}
\|u\|_{C^{\gamma ; \frac{\gamma}{2}}\left(\bar{U} \times\left[\tau_{1}, \tau_{2}\right]\right)} & \leq \sum_{i=1}^{N}\left\|\phi_{i} u\right\|_{C^{\gamma ; \frac{\gamma}{2}}\left(\bar{U} \times\left[\tau_{1}, \tau_{2}\right]\right)} \leq \sum_{i=1}^{N}\left\|\phi_{i} u\right\|_{C^{\gamma ; \frac{\gamma}{2}}(\bar{Q})} \\
& \leq \sum_{i=1}^{N}\left\|\phi_{i}\right\|_{C^{\gamma ; \frac{\gamma}{2}(\bar{Q})}\|u\|_{C^{\gamma ; \frac{\gamma}{2}}\left(\bar{R}_{r}\left(x_{i}\right) \times\left[t_{i}-r^{2}, t_{i}\right]\right)}} \\
& \leq \underbrace{\left(\sum_{i=1}^{N}\left\|\phi_{i}\right\|_{C^{\gamma ; \frac{\gamma}{2}(\bar{Q})}} \cdot\left(1+C_{1} r^{-\gamma}\right)\right)}_{=: C_{2}} \cdot \max _{1 \leq i \leq N}\left(\sup _{R_{x_{i}(3 r)} \times\left(t_{i}-(3 r)^{2}, t_{i}\right)} u\right) .
\end{aligned}
$$


Then, by [2, Theorems 2 and 3], for each $1 \leq i \leq N$

$$
\begin{aligned}
\sup _{R_{x_{i}}(3 r) \times\left(t_{i}-(3 r)^{2}, t_{i}\right)} u & \leq C_{3}\|u\|_{L^{2}\left(R_{x_{i}(9 r)} \times\left(t_{i}-(9 r)^{2}, t_{i}\right)\right)} \\
& \leq C_{3}(18 r)^{\frac{d}{2}} \cdot(9 r) \sup _{R_{x_{i}}(9 r) \times\left(t_{i}-(9 r)^{2}, t_{i}\right)} u \\
& \leq C_{3}(18 r)^{\frac{d}{2}} \cdot(9 r) \cdot C_{4} \inf _{R_{x_{i}}(9 r) \times\left(t_{i}+6(9 r)^{2}, t_{i}+7(9 r)^{2}\right)} u \\
& \leq C_{3} C_{4}(18 r)^{-\frac{d}{2}} \cdot(9 r)^{-1}\|u\|_{L^{1}\left(R_{x_{i}}(9 r) \times\left(t_{i}+6(9 r)^{2}, t_{i}+7(9 r)^{2}\right)\right)} \\
& \leq \underbrace{C_{3} C_{4}(18 r)^{-\frac{d}{2}}(9 r)^{-1}}_{=: C_{5}}\|u\|_{L^{1}\left(V \times\left(\tau_{3}, \tau_{4}\right)\right)}
\end{aligned}
$$

where $C_{3}$ and $C_{4}$ are constants which are independent of $f$ and $x_{i}$. Combining (28), (29) we have for $s \in[1, \infty)$

$$
\begin{aligned}
\|P . f(\cdot)\|_{C^{\gamma ; \frac{\gamma}{2}}\left(\bar{U} \times\left[\tau_{1}, \tau_{2}\right]\right)} & \leq\left\|\rho^{-1}\right\|_{C^{\gamma}\left(\bar{U} \times\left[\tau_{1}, \tau_{2}\right]\right)}\|\rho(\cdot) P . f(\cdot)\|_{C^{\gamma ; \frac{\gamma}{2}}\left(\bar{U} \times\left[\tau_{1}, \tau_{2}\right]\right)} \\
& \leq \underbrace{\left\|\rho^{-1}\right\|_{C^{\gamma}\left(\bar{U} \times\left[\tau_{1}, \tau_{2}\right]\right)} C_{2} C_{5}}_{=: C_{6}}\|P . f(\cdot)\|_{L^{1}\left(V \times\left(\tau_{3}, \tau_{4}\right), m \otimes d t\right)} \\
& \leq C_{6}\left(\tau_{4}-\tau_{3}\right)\|\rho\|_{L^{1}(V)}^{\frac{s-1}{s}}\|f\|_{L^{s}\left(\mathbb{R}^{d}, m\right)} .
\end{aligned}
$$

For $f \in L^{s}\left(\mathbb{R}^{d}, m\right), f \geq 0$, define

$$
P . f(\cdot):=\lim _{n \rightarrow \infty} P . f_{n}(\cdot) \text { in } C^{\gamma ; \frac{\gamma}{2}}\left(\bar{U} \times\left[\tau_{1}, \tau_{2}\right]\right),
$$

where $\left(f_{n}\right)_{n \geq 1} \subset C_{0}^{\infty}\left(\mathbb{R}^{d}\right)$ is any sequence of positive functions converging to $f$ in $L^{s}\left(\mathbb{R}^{d}, m\right)$. Then P.f(.) is well-defined, i.e. independent of the choice of $\left(f_{n}\right)_{n \geq 1}$, and (30) (including all intermediate inequalities) extends to $f \in L^{s}\left(\mathbb{R}^{d}, m\right), f \geq 0$. In particular, (26) holds for $f \in$ $L^{s}\left(\mathbb{R}^{d}, m\right), f \geq 0, s \in[1, \infty)$.

Moreover, given $f \in L^{s}\left(\mathbb{R}^{d}, m\right), f \geq 0$, and $f_{n} \in C_{0}^{\infty}\left(\mathbb{R}^{d}\right), f_{n} \geq 0$, with $f_{n} \rightarrow f$ in $L^{s}\left(\mathbb{R}^{d}, m\right)$, for each $t>0$ we have $T_{t} f_{n} \rightarrow T_{t} f$ in $L^{s}(U, m)$ and also $P_{t} f_{n} \rightarrow P_{t} f$ in $L^{s}(U, m)$ by (31) holds for $s \in[1, \infty)$. Thus

$$
P_{t} f=T_{t} f \quad m \text {-a.e. on } U \text { for each } t>0 .
$$

This holds for arbitrary bounded open $U$, hence also on $\mathbb{R}^{d}$. Thus $P_{t} f$ is an $m$-version of $T_{t} f$. For $f \in L^{\infty}\left(\mathbb{R}^{d}, m\right), f \geq 0$, take $f_{n}:=1_{B_{n}} \cdot f$ with $n \geq 1$. Then for each $t>0$,

$$
T_{t} f=\lim _{n \rightarrow \infty} T_{t} f_{n}=\lim _{n \rightarrow \infty} P_{t} f_{n}, m \text {-a.e. on } \mathbb{R}^{d} .
$$

For each fixed $(x, t) \in V \times\left(\tau_{3}, \tau_{4}\right),\left(P_{t} f_{n}(x)\right)_{n \geq 1}$ is an increasing sequence of real numbers that is bounded by one by the sub-Markovian property and continuity of $z \mapsto P_{t} f_{n}(z)$. Thus (26) for $s=1$ and Lebesgue's dominated convergence theorem imply that $\left(P . f_{n}(\cdot)\right)_{n \geq 1}$ is a Cauchy sequence in $C^{\gamma ; \frac{\gamma}{2}}\left(\bar{U} \times\left[\tau_{1}, \tau_{2}\right]\right)$. Hence we can again define

$$
P . f(\cdot):=\lim _{n \rightarrow \infty} P . f_{n}(\cdot) \text { in } C^{\gamma ; \frac{\gamma}{2}}\left(\bar{U} \times\left[\tau_{1}, \tau_{2}\right]\right)
$$

and (26) also holds for $s=\infty$. Moreover for each $t>0, P_{t} f_{n}$ converges uniformly to $P_{t} f$ in $U$, hence in view of (33), (32) also holds for $s=\infty$. Since $U$ is an arbitrary bounded open subset in 
$\mathbb{R}^{d}$, we have hence shown that for any $f \in \cup_{s \in[1, \infty]} L^{s}\left(\mathbb{R}^{d}, m\right), f \geq 0, P$. $f(\cdot)$ is locally parabolic Hölder continuous in $\mathbb{R}^{d} \times(0, \infty)$ and for each $t>0, P_{t} f=T_{t} f m$-a.e. on $\mathbb{R}^{d}$. By linearity the latter is obviously also true for $f$ without the positivity assumption.

Remark 3.9 (i) (26) easily implies for any $s \in[1, \infty], f \in L^{s}\left(\mathbb{R}^{d}, m\right), t>0$ (cf. for instance (30) for $s \in[0, \infty)$ and use the sub-Markovian property for $s=\infty$ ) that

$$
\left\|P_{t} f\right\|_{C^{0, \gamma}(\bar{U})} \leq 2 C_{6}\left(\tau_{4}-\tau_{3}\right)\|\rho\|_{L^{1}(V)}^{\frac{s-1}{s}} \cdot\|f\|_{L^{s}\left(\mathbb{R}^{d}, m\right)},
$$

where $\frac{s-1}{s}:=1$ for $s=\infty$. (34) is an improvement over (23) in regard to analyticity, which is no more required for (34), and in regard to the integrability order which is $s \in[1, \infty]$ for (34) but $r \in[q \vee 2, \infty)$ for (23). Only the Hölder exponent $\gamma$ in (34) depends on the domain and may vary, whereas in (23) it is always $\beta$ as in (16), independently of the domain.

Using Theorem 3.8, we can define $P_{t}(x, A)$ as in (24) and we see that there exist unique subprobability measures $P_{t}(x, d y)$ on $\left(\mathbb{R}^{d}, \mathcal{B}\left(\mathbb{R}^{d}\right)\right)$, absolutely continuous with respect to $m$ and with Radon-Nikodym derivatives $p_{t}(x, \cdot)$ defined by (25).

(ii) Let $A:=\left(a_{i j}\right)_{1 \leq i, j \leq d}, \mathbf{G}, \rho, \beta^{\rho, A}$, and $\mathbf{B}$ be as in Theorem [3.2, but suppose $p>d+$ 2 and that $m$ is a probability measure. In this case similar results to Theorem 3.8 and the following Proposition 3.10(ii) and some additional structure with respect to duality is derived in [6. Theorem 4.1]. The technique of proof is different to ours but also applies if $m$ is not restricted to be a probability measure (cf. [6, Remark 4.2(ii)]). However, we insist that $K_{t}(x, d y)$ as occurring in [6, Remark 4.2(ii)] is in contrast to what is mentioned in [6, Remark 4.2(ii)] always a sub-probability measure and hence finite and moreover in case of merely locally finite measure only the $L^{1}\left(\mathbb{R}^{d}, m\right)$-strong Feller property follows, whereas we derive the $L^{[1, \infty]}\left(\mathbb{R}^{d}, m\right)$ strong Feller property (see Theorem 3.8 and Proposition 3.10 for the definition), that includes the classical strong Feller property.

(iii) As opposed to [1, Proposition 3.8], we do not need the condition $\alpha R_{\alpha} 1_{\mathbb{R}^{d}} \equiv 1$ in order to derive the classical strong Feller property of $\left(P_{t}\right)_{t>0}$. Also in [39], non-explosion (see (49) below) is used to obtain the classical strong Feller property.

Using Theorem 3.8, we obtain the following improvement of Proposition 3.6.

Proposition 3.10 Let $t, \alpha>0$ be arbitrary. Let $q, \beta$ be defined as in (12), (16), $r_{\alpha}(x, y)$ as in Remark 3.7, and $p_{t}(x, y)$ as in Remark 3.9. Then under the conditions of Theorem 3.2, it holds:

(i) $G_{\alpha} g$ has a locally Hölder continuous m-version of order $\beta=1-d / p$

$$
R_{\alpha} g=\int_{\mathbb{R}^{d}} g(y) R_{\alpha}(\cdot, d y)=\int_{\mathbb{R}^{d}} g(y) r_{\alpha}(\cdot, y) m(d y), \quad \forall g \in \bigcup_{r \in[q, \infty]} L^{r}\left(\mathbb{R}^{d}, m\right) .
$$

In particular, (35) extends by linearity to all $g \in L^{q}\left(\mathbb{R}^{d}, m\right)+L^{\infty}\left(\mathbb{R}^{d}, m\right)$, i.e. $\left(R_{\alpha}\right)_{\alpha>0}$ is $L^{[q, \infty]}\left(\mathbb{R}^{d}, m\right)$-strong Feller.

(ii) $T_{t} f$ has a continuous m-version

$$
P_{t} f=\int_{\mathbb{R}^{d}} f(y) P_{t}(\cdot, d y)=\int_{\mathbb{R}^{d}} f(y) p_{t}(\cdot, y) m(d y), \quad \forall f \in \bigcup_{s \in[1, \infty]} L^{s}\left(\mathbb{R}^{d}, m\right) .
$$


( $P_{t} f$ is locally Hölder continuous of order $\beta=1-d / p$, if $f \in \bigcup_{r \in[q, \infty)} D\left(L_{r}\right)$ ) and locally Hölder continuous with possibly changing Hölder exponents, if $f \in \bigcup_{s \in[1, \infty]} L^{s}\left(\mathbb{R}^{d}, m\right) \backslash$ $\bigcup_{r \in[q, \infty)} D\left(L_{r}\right)$. In particular, (36) extends by linearity to all $f \in L^{1}\left(\mathbb{R}^{d}, m\right)+L^{\infty}\left(\mathbb{R}^{d}, m\right)$, i.e. $\left(P_{t}\right)_{t>0}$ is $L^{[1, \infty]}\left(\mathbb{R}^{d}, m\right)$-strong Feller.

Finally, for any $\alpha>0, x \in \mathbb{R}^{d}, g \in L^{q}\left(\mathbb{R}^{d}, m\right)+L^{\infty}\left(\mathbb{R}^{d}, m\right)$

$$
R_{\alpha} g(x)=\int_{0}^{\infty} e^{-\alpha t} P_{t} g(x) d t
$$

Proof Fix $\alpha>0, t>0, x \in \mathbb{R}^{d}$. Let $A \in \mathcal{B}\left(\mathbb{R}^{d}\right)$. Using (21), (22), monotone integration and (18), we can see that

$$
\int_{\mathbb{R}^{d}} 1_{A}(y) r_{\alpha}(x, y) m(d y)=\int 1_{A}(y) R_{\alpha}(x, d y)=\lim _{l \rightarrow \infty} R_{\alpha}\left(1_{B_{l} \cap A}\right)(x)=R_{\alpha} 1_{A}(x) .
$$

Using (24), (25), monotone integration and (26) (cf. proof of Theorem 3.8), we can see that

$$
\int_{\mathbb{R}^{d}} 1_{A}(y) p_{t}(x, y) m(d y)=\int_{\mathbb{R}^{d}} 1_{A}(y) P_{t}(x, d y)=\lim _{l \rightarrow \infty} P_{t} 1_{B_{l} \cap A}(y)=P_{t} 1_{A}(x) .
$$

(37), resp. (38) extends to $g \in L^{r}\left(\mathbb{R}^{d}, m\right), r \in[q, \infty]$, resp. $g \in L^{s}\left(\mathbb{R}^{d}, m\right), s \in[1, \infty]$ in the following way. Split $g, f$ in positive and negative parts. We may hence assume that $g, f$ are positive. Then we use a monotone approximation of $g$, resp. $f$ with simple functions involving indicator functions like above, i.e. there exists an increasing sequence of simple functions $\left(g_{n}\right)_{n \geq 1}$ with $0 \leq g_{n} \nearrow g$, resp. $\left(f_{n}\right)_{n \geq 1}$ with $0 \leq f_{n} \nearrow f$. By this we can use monotone integration for the two left hand terms of (37), resp. (38), and (18), resp. (26) for the left hand term. Thus (i) and (ii) follow.

The last statement follows similarly noting that for $A \in \mathcal{B}\left(\mathbb{R}^{d}\right)$

$$
R_{\alpha} 1_{A}=\int_{0}^{\infty} e^{-\alpha t} P_{t} 1_{A} d t
$$

$m$-a.e. hence everywhere since both sides define continuous functions and we can as before use monotone integration as well as (18) and (26) to prove the remaining assertion.

Remark 3.11 Under the conditions of Theorem [3.2, we obtain analogously to [1] that $\left(P_{t}\right)_{t>0}$ defined on

$$
L^{\infty}\left(\mathbb{R}^{d}, m\right)=L^{\infty}\left(\mathbb{R}^{d}\right) \supset \mathcal{B}_{b}\left(\mathbb{R}^{d}\right)
$$

determines a (temporally homogeneous) submarkovian transition function (cf. [10, 1.2]). Thus $\left(P_{t}\right)_{t>0}$ satisfies condition $(\mathbf{H 1})$ of [28]. Moreover, $P_{t} f, t>0$, is by Proposition [3.10(ii) independent of the $m$-version chosen for $f \in L^{\infty}\left(\mathbb{R}^{d}, m\right)$.

By the results of [33, Section 4.1], the generalized Dirichlet form $\mathcal{E}$ associated with $\left(L_{2}, D\left(L_{2}\right)\right)$ is strictly quasi-regular. In particular, by [33, Theorem 6] there exists a Hunt process

$$
\tilde{\mathbb{M}}=\left(\tilde{\Omega}, \tilde{\mathcal{F}},(\tilde{\mathcal{F}})_{t \geq 0},\left(\tilde{X}_{t}\right)_{t \geq 0},\left(\tilde{\mathbb{P}}_{x}\right)_{x \in \mathbb{R}^{d} \cup\{\Delta\}}\right)
$$


with state space $\mathbb{R}^{d}$ and life time $\tilde{\zeta}:=\inf \left\{t \geq 0: \tilde{X}_{t}=\Delta\right\}$ and cemetery $\Delta$ such that $\mathcal{E}$ is (strictly properly) associated with $\tilde{\mathbb{M}}$.

For some fixed $\varphi \in L^{1}\left(\mathbb{R}^{d}, m\right)_{b}, 0<\varphi \leq 1$, consider the strict capacity cap ${ }_{1, \widehat{G}_{1} \varphi}$ of $\mathcal{E}$ as defined in [33, Definition 1]. Due to the properties of smooth measures with respect to $\operatorname{cap}_{1, \widehat{G}_{1} \varphi}$ in $[33$, Section 3] one can consider the work [34] with $\operatorname{cap}_{\varphi}$ (as defined in [34]) replaced by $\operatorname{cap}_{1, \widehat{G}_{1 \varphi}}$. In particular [34, Theorem 3.10 and Proposition 4.2] apply with respect to the strict capacity $\operatorname{cap}_{1, \widehat{G}_{1} \varphi}$ and therefore the paths of $\tilde{\mathbb{M}}$ are continuous $\tilde{\mathbb{P}}_{x}$-a.s. for strictly $\mathcal{E}$-q.e. $x \in \mathbb{R}^{d}$ on the one-point-compactification $\mathbb{R}_{\Delta}^{d}$ of $\mathbb{R}^{d}$ with $\Delta$ as point at infinity. We may hence assume that

$$
\tilde{\Omega}=\left\{\omega=(\omega(t))_{t \geq 0} \in C\left([0, \infty), \mathbb{R}_{\Delta}^{d}\right): \omega(t)=\Delta \quad \forall t \geq \zeta(\omega)\right\}
$$

and

$$
\tilde{X}_{t}(\omega)=\omega(t), \quad t \geq 0 .
$$

Now, we can apply the Dirichlet form method of [28, Section 2.1.2]. There it was only developed in a symmetric setting. But here we are in the non-sectorial setting. However one can readily check that it works nearly in the same way using Lemma 3.1 instead of [28, Lemma 2.5(i)] and modifying (H2)' of [28, Section 2.1.2] in the following way:

(H2) $)^{\prime}$ We can find $\left\{u_{n}: n \geq 1\right\} \subset D\left(L_{1}\right) \cap C_{0}\left(\mathbb{R}^{d}\right)$ satisfying:

(i) For all $\varepsilon \in \mathbb{Q} \cap(0,1)$ and $y \in D$, where $D$ is any given countable dense set in $\mathbb{R}^{d}$, there exists $n \in \mathbb{N}$ such that $u_{n}(z) \geq 1$, for all $z \in \bar{B}_{\frac{\varepsilon}{4}}(y)$ and $u_{n} \equiv 0$ on $\mathbb{R}^{d} \backslash B_{\frac{\varepsilon}{2}}(y)$,

(ii) $R_{1}\left(\left[\left(1-L_{1}\right) u_{n}\right]^{+}\right), R_{1}\left(\left[\left(1-L_{1}\right) u_{n}\right]^{-}\right), R_{1}\left(\left[\left(1-L_{1}\right) u_{n}^{2}\right]^{+}\right), R_{1}\left(\left[\left(1-L_{1}\right) u_{n}^{2}\right]^{-}\right)$are continuous on $\mathbb{R}^{d}$ for all $n \geq 1$,

and

(iii) $R_{1} C_{0}\left(\mathbb{R}^{d}\right) \subset C\left(\mathbb{R}^{d}\right)$,

(iv) For any $f \in C_{0}\left(\mathbb{R}^{d}\right)$ and $x \in \mathbb{R}^{d}$, the map $t \mapsto P_{t} f(x)$ is right-continuous on $(0, \infty)$.

We have $C_{0}^{2}\left(\mathbb{R}^{d}\right) \subset D\left(L_{1}\right) \cap C_{0}\left(\mathbb{R}^{d}\right)$ and moreover obviously $\left(1-L_{1}\right) u,\left(1-L_{1}\right) u^{2} \in L^{p}\left(\mathbb{R}^{d}\right)_{0}$ for any $u \in C_{0}^{2}\left(\mathbb{R}^{d}\right)$. Consequently, by Theorem 3.8 and Proposition 3.10, $(\mathbf{H 2})^{\prime}$ is satisfied for some countable subset of $C_{0}^{2}\left(\mathbb{R}^{d}\right)$.

Therefore, we obtain:

Theorem 3.12 Under the conditions of Theorem 3.2, there exists a Hunt process

$$
\mathbb{M}=\left(\Omega, \mathcal{F},\left(\mathcal{F}_{t}\right)_{t \geq 0},\left(X_{t}\right)_{t \geq 0},\left(\mathbb{P}_{x}\right)_{x \in \mathbb{R}^{d} \cup\{\Delta\}}\right)
$$

with state space $\mathbb{R}^{d}$ and life time

$$
\zeta=\inf \left\{t \geq 0: X_{t}=\Delta\right\}=\inf \left\{t \geq 0: X_{t} \notin \mathbb{R}^{d}\right\},
$$

having the transition function $\left(P_{t}\right)_{t \geq 0}$ as transition semigroup, such that $\mathbb{M}$ has continuous sample paths in the one point compactification $\mathbb{R}_{\Delta}^{d}$ of $\mathbb{R}^{d}$ with the cemetery $\Delta$ as point at infinity.

Remark 3.13 Checking the details of [1, Section 4] one by one with possibly only few modifications one may possibly also obtain Theorem 3.12. 
Lemma 3.14 Let $\mathbb{E}_{x}$ denote the expectation with respect to $\mathbb{P}_{x}, x \in \mathbb{R}^{d}$, where $\mathbb{M}$ is as in Theorem 3.12. Then:

(i) For any $x \in \mathbb{R}^{d}, \alpha>0, t>0$, we have

$$
R_{\alpha} g(x)=\int_{\mathbb{R}^{d}} r_{\alpha}(x, y) g(y) m(d y)=\mathbb{E}_{x}\left[\int_{0}^{\infty} e^{-\alpha s} g\left(X_{s}\right) d s\right]
$$

for any $g \in L^{q}\left(\mathbb{R}^{d}, m\right)+L^{\infty}\left(\mathbb{R}^{d}, m\right)$, and

$$
P_{t} f(x)=\int_{\mathbb{R}^{d}} p_{t}(x, y) f(y) m(d y)=\mathbb{E}_{x}\left[f\left(X_{t}\right)\right]
$$

for any $f \in L^{1}\left(\mathbb{R}^{d}, m\right)+L^{\infty}\left(\mathbb{R}^{d}, m\right)$.

In particular, integrals of the form $\int_{0}^{\infty} e^{-\alpha s} h\left(X_{s}\right) d s, \int_{0}^{t} h\left(X_{s}\right) d s, t \geq 0$ are for any $x \in \mathbb{R}^{d}$, whenever they are well-defined, $\mathbb{P}_{x}$-a.s. independent of the measurable $m$-version chosen for $h$.

(ii) Let $g \in L^{r}\left(\mathbb{R}^{d}, m\right)$ for some $r \in[q, \infty]$. Then for any ball $B$ there exists a constant $c_{B, r}$, depending in particular on $B$ and $r$, such that for all $t \geq 0$

$$
\sup _{x \in \bar{B}} \mathbb{E}_{x}\left[\int_{0}^{t}|g|\left(X_{s}\right) d s\right] \leq e^{t} c_{B, r}\|g\|_{L^{r}\left(\mathbb{R}^{d}, m\right)} .
$$

In particular, by the local boundedness of $\rho$, for any $f \in L^{q}\left(\mathbb{R}^{d}\right)_{0}$, and any open ball $B$, (40) implies

$$
\sup _{x \in \bar{B}} \mathbb{E}_{x}\left[\int_{0}^{t}|f|\left(X_{s}\right) d s\right] \leq c\|f\|_{L^{q}\left(\mathbb{R}^{d}\right)},
$$

where the constant $c$ depends only on $t, q, B$ and the support of $f$.

(iii) Let $u \in D\left(L_{r}\right)$, for some $r \in[q, \infty)$ and $\alpha>0, t>0$. Then for any $x \in \mathbb{R}^{d}$

$$
R_{\alpha}\left(\left(\alpha-L_{r}\right) u\right)(x)=u(x),
$$

and

$$
P_{t} u(x)-u(x)=\int_{0}^{t} P_{s}\left(L_{r} u\right)(x) d s .
$$

Proof (i) By Remark 3.11 and Theorem 3.12, we have for any $t>0, x \in \mathbb{R}^{d}, h \in L^{\infty}\left(\mathbb{R}^{d}, m\right)$

$$
P_{t} h(x)=\int_{\mathbb{R}^{d}} p_{t}(x, y) h(y) m(d y)=\mathbb{E}_{x}\left[h\left(X_{t}\right)\right],
$$

and the expressions in (41) are all well-defined, i.e. do not change in value for any $m$-version of $h$. Now the resolvent and semigroup representations follow by splitting functions in $g \in$ $\bigcup_{r \in[q, \infty]} L^{r}\left(\mathbb{R}^{d}, m\right)$ and $f \in \bigcup_{s \in[1, \infty]} L^{s}\left(\mathbb{R}^{d}, m\right)$ into their positive and negative parts, using monotone approximations of these with functions in $L^{\infty}\left(\mathbb{R}^{d}, m\right)$ and finally linearity, which is possible since all expressions are finite by Proposition 3.10. In particular, the limits will as the 
original expressions in (41) also not depend on the chosen $m$-versions, which concludes the proof. (ii) Using in particular (i) and (19), we get

$$
\begin{aligned}
\sup _{x \in \bar{B}} \mathbb{E}_{x}\left[\int_{0}^{t}|g|\left(X_{s}\right) d s\right] & \leq e^{t} \sup _{x \in \bar{B}} \mathbb{E}_{x}\left[\int_{0}^{\infty} e^{-s}|g|\left(X_{s}\right) d s\right] \\
& =e^{t} \sup _{x \in \bar{B}} R_{1}|g|(x) \leq e^{t} c_{B}\|g\|_{L^{r}\left(\mathbb{R}^{d}, m\right)} .
\end{aligned}
$$

Using (i), the proof of (iii) works exactly as in [1, Lemma 5.1]. However, we emphasize that due to the increased regularity $r \geq q$ from (i) (coming from Proposition 3.6) in comparison to $r \geq p$ in [1], we obtain more general statements in (ii) and (iii).

For $A \in \mathcal{B}\left(\mathbb{R}^{d}\right)$, define

$$
\sigma_{A}:=\inf \left\{t>0: X_{t} \in A\right\}
$$

and

$$
\sigma_{n}:=\sigma_{\mathbb{R}^{d} \backslash B_{n}}, n \geq 1
$$

Lemma 3.15 Let $\mathbb{M}$ be as in Theorem 3.12, Then:

(i) For any $x \in \mathbb{R}^{d}$, we have

$$
\mathbb{P}_{x}\left(\lim _{n \rightarrow \infty} \sigma_{n} \geq \zeta\right)=1
$$

(ii) For any $x \in \mathbb{R}^{d}, t \geq 0$, we have

$$
\mathbb{P}_{x}\left(\int_{0}^{t}|f|\left(X_{s}\right) d s<\infty\right)=1 \text {, if } f \in \bigcup_{r \in[q, \infty]} L^{r}\left(\mathbb{R}^{d}, m\right)
$$

and

$$
\mathbb{P}_{x}\left(\left\{\int_{0}^{t}|f|\left(X_{s}\right) d s<\infty\right\} \cap\{t<\zeta\}\right)=\mathbb{P}_{x}(\{t<\zeta\}) \text {, if } \quad f \in L_{l o c}^{q}\left(\mathbb{R}^{d}, m\right)
$$

Proof (i) By Proposition 3.10 and Lemma 3.14(i), we have that $\mathbb{E}$. $\left[\int_{0}^{\infty} e^{-\alpha s} g\left(X_{s}\right) d s\right]$ is an $m$-version of $G_{\alpha} g$, for all $\alpha>0$ and $g \in L^{\infty}\left(\mathbb{R}^{d}, m\right)$. It hence follows by [29, IV. Theorem 3.1] (or [33, Proposition 2(ii)]) that $\mathcal{E}$ is quasi-regular. Therefore by [29, IV. Definition 1.7] there exists an $\mathcal{E}$-nest $\left(E_{k}\right)_{k \geq 1}$ of compact subsets of $\mathbb{R}^{d}$. Then [29, IV. Lemma 3.10] implies, $\mathbb{P}_{x}\left(\lim _{k \rightarrow \infty} \sigma_{\mathbb{R}^{d} \backslash E_{k}} \geq \zeta\right)=1$ for $\mathcal{E}$-q.e. $x \in \mathbb{R}^{d}$, hence in particular for $m$-a.e. $x \in \mathbb{R}^{d}$ by [29, III. Remark 2.6]. Since $\left(B_{n}\right)_{n \geq 1}$ is an open cover of $E_{k}$ for each $k$, and $\sigma_{A} \leq \sigma_{B}$ whenever $B \subset A$, we then obtain $\mathbb{P}_{x}\left(\lim _{n \rightarrow \infty} \sigma_{n} \geq \zeta\right)=1$ for $m$-a.e. $x \in \mathbb{R}^{d}$. Now the result follows exactly as in [27. Lemma 3.3].

(ii) The first statement immediately follows from Lemma 3.14(ii). For the second statement it is enough to show that for any $t \geq 0$ and $x \in \mathbb{R}^{d}$

$$
\mathbb{P}_{x}\left(1_{\{t<\zeta\}} \int_{0}^{t}|f|\left(X_{s}\right) d s<\infty\right)=1, \text { if } \quad f \in L_{l o c}^{q}\left(\mathbb{R}^{d}, m\right)
$$


It holds $\mathbb{P}_{x}\left(n \wedge \sigma_{n}<\zeta\right)=1$ for any $n \geq 1$ and $x \in \mathbb{R}^{d}$, since $\mathbb{M}$ has continuous sample paths on the one-point-compactification $\mathbb{R}_{\Delta}^{d}$. Thus using (i), we get that the left hand side of (42) equals

$$
\lim _{n \rightarrow \infty} \mathbb{P}_{x}\left(1_{\left\{t<n \wedge \sigma_{n}\right\}} \int_{0}^{t}|f|\left(X_{s}\right) d s<\infty\right)
$$

Now, fix $x \in \mathbb{R}^{d}$. Then there exists $N_{0} \in \mathbb{N}$ with $x \in B_{n}$ for any $n \geq N_{0}$. Consequently, for any $n \geq N_{0}$ we have $\mathbb{P}_{x}$-a.s. that $X_{s} \in B_{n}$ for any $s \in[0, t]$, if $t<\sigma_{n}$. It follows with the help of Lemma 3.14 (ii)

$$
\mathbb{E}_{x}\left[1_{\left\{t<n \wedge \sigma_{n}\right\}} \int_{0}^{t}|f|\left(X_{s}\right) d s\right] \leq \mathbb{E}_{x}\left[\int_{0}^{t}|f| 1_{B_{n}}\left(X_{s}\right) d s\right]<\infty, \quad \forall n \geq N_{0} .
$$

Thus each sequence member in (43) is equal to one and therefore (42) holds.

Proposition 3.16 Let $\mathbb{M}$ be as in Theorem 3.12. Let $u \in D\left(L_{r}\right)$, for some $r \in[q, \infty)$. Then

$$
M_{t}^{u}:=u\left(X_{t}\right)-u(x)-\int_{0}^{t} L_{r} u\left(X_{s}\right) d s, \quad t \geq 0,
$$

is a continuous $\left(\mathcal{F}_{t}\right)_{t \geq 0}$-martingale under $\mathbb{P}_{x}$ for any $x \in \mathbb{R}^{d}$. If $r \geq 2 q$, then $M^{u}$ is square integrable.

Proof The first result is an immediate consequence of Lemma 3.14 (see for instance [12, Chapter 7, (1.6) Theorem]). The second follows from Lemma 3.14(i) and (ii).

Proposition 3.17 Let $\mathbb{M}$ be as in Theorem 3.12. Let $u \in C_{0}^{2}\left(\mathbb{R}^{d}\right), t \geq 0$. Then the quadratic variation process $\left\langle M^{u}\right\rangle$ of the continuous martingale $M^{u}$ satisfies $\mathbb{P}_{x}$-a.s for any $x \in \mathbb{R}^{d}, t \geq 0$

$$
\left\langle M^{u}\right\rangle_{t}=\int_{0}^{t}\langle A \nabla u, \nabla u\rangle\left(X_{s}\right) d s
$$

In particular, by Lemma 3.14(ii) $\left\langle M^{u}\right\rangle_{t}$ is $\mathbb{P}_{x}$-integrable for any $x \in \mathbb{R}^{d}, t \geq 0$ and so $M^{u}$ is square integrable.

Proof For $g \in C_{0}^{2}\left(\mathbb{R}^{d}\right)$, we have $g \in D\left(L_{r}\right)$ and $L_{1} g=L_{r} g$ for any $r \in[1, p]$. Thus for $u \in C_{0}^{2}\left(\mathbb{R}^{d}\right)$, we get by Proposition 3.16 and Lemma 3.1

$$
u^{2}\left(X_{t}\right)-u^{2}(x)=M_{t}^{u^{2}}+\int_{0}^{t}\left(\langle A \nabla u, \nabla u\rangle\left(X_{s}\right)+2 u L_{1} u\left(X_{s}\right)\right) d s .
$$

Applying Itô's formula to the continuous semimartingale $\left(u\left(X_{t}\right)\right)_{t \geq 0}$, we obtain

$$
u^{2}\left(X_{t}\right)-u^{2}(x)=\int_{0}^{t} 2 u\left(X_{s}\right) d M_{s}^{u}+\int_{0}^{t} 2 u L_{r} u\left(X_{s}\right) d s+\left\langle M^{u}\right\rangle_{t} .
$$

The last two equalities imply that $\left(\left\langle M^{u}\right\rangle_{t}-\int_{0}^{t}\langle A \nabla u, \nabla u\rangle\left(X_{s}\right) d s\right)_{t \geq 0}$ is a continuous $\mathbb{P}_{x^{-}}$martingale of bounded variation for any $x \in \mathbb{R}^{d}$. This implies the assertion.

For the following result, see for instance [9, Theorem 1.1, Lemma 2.1], that we can apply locally. 
Lemma 3.18 Under the assumptions of Theorem 3.2 on the diffusion matrix $A$, there exists a matrix of functions $\sigma=\left(\sigma_{i j}\right)_{1 \leq i, j \leq d}$ with $\sigma_{i j} \in C\left(\mathbb{R}^{d}\right)$ for all $i, j$ such that

$$
A(x)=\sigma \sigma^{T}(x), \quad \forall x \in \mathbb{R}^{d},
$$

i.e.

$$
a_{i j}(x)=\sum_{k=1}^{d} \sigma_{i k}(x) \sigma_{j k}(x), \quad \forall x \in \mathbb{R}^{d}, 1 \leq i, j \leq d
$$

and

$$
\operatorname{det}(\sigma(x))>0, \quad \forall x \in \mathbb{R}^{d}
$$

Theorem 3.19 Let $A:=\left(a_{i j}\right)_{1 \leq i, j \leq d}, \mathbf{G}$, be as in Theorem 3.2. Consider the Hunt process $\mathbb{M}$ from Theorem 3.12 with coordinates $X_{t}=\left(X_{t}^{1}, \ldots, X_{t}^{d}\right)$ and suppose that $\mathbb{M}$ is non-explosive, i.e.

$$
\mathbb{P}_{x}(\zeta=\infty)=1 \text { for any } x \in \mathbb{R}^{d} .
$$

(i) Let $\left(\sigma_{i j}\right)_{1 \leq i, j \leq d}$ be as in Lemma [3.18. Then it holds $\mathbb{P}_{x}$-a.s. for any $x=\left(x_{1}, \ldots, x_{d}\right) \in \mathbb{R}^{d}$, $i=1, \ldots, d$

$$
X_{t}^{i}=x_{i}+\sum_{j=1}^{d} \int_{0}^{t} \sigma_{i j}\left(X_{s}\right) d W_{s}^{j}+\int_{0}^{t} g_{i}\left(X_{s}\right) d s, \quad 0 \leq t<\infty,
$$

where $W=\left(W^{1}, \ldots, W^{d}\right)$ is a standard d-dimensional Brownian motion starting from zero.

(ii) Let $\left(\sigma_{i j}\right)_{1 \leq i \leq d, 1 \leq j \leq m}, m \in \mathbb{N}$ arbitrary but fixed, be any matrix consisting of continuous functions $\sigma_{i j} \in C\left(\mathbb{R}^{d}\right)$ for all $i, j$, such that $A=\sigma \sigma^{T}$ (where $A$ satisfies the assumptions of Theorem [3.2), i.e.

$$
a_{i j}(x)=\sum_{k=1}^{m} \sigma_{i k}(x) \sigma_{j k}(x), \quad \forall x \in \mathbb{R}^{d}, 1 \leq i, j \leq d .
$$

Then on a standard extension of $\left(\Omega, \mathcal{F},\left(\mathcal{F}_{t}\right)_{t \geq 0}, \mathbb{P}_{x}\right), x \in \mathbb{R}^{d}$, that we denote for notational convenience again by $\left(\Omega, \mathcal{F},\left(\mathcal{F}_{t}\right)_{t \geq 0}, \mathbb{P}_{x}\right), x \in \mathbb{R}^{d}$, there exists a standard $m$-dimensional Brownian motion $W=\left(W^{1}, \ldots, W^{m}\right)$ starting from zero such that 44) holds with $\sum_{j=1}^{d}$ replaced by $\sum_{j=1}^{m}$.

Proof (i) Consider the stopping times

$$
D_{n}:=D_{\mathbb{R}^{d} \backslash B_{n}}:=\inf \left\{t \geq 0: X_{t} \in \mathbb{R}^{d} \backslash B_{n}\right\} \quad n \geq 1 .
$$

Since $\mathbb{M}$ is non-explosive, it follows from Lemma 3.15(i) that $D_{n} \nearrow \infty \mathbb{P}_{x^{-}}$a.s. for any $x \in \mathbb{R}^{d}$. Let $v \in C^{2}\left(\mathbb{R}^{d}\right)$. Then we claim that

$$
M_{t}^{v}:=v\left(X_{t}\right)-v(x)-\int_{0}^{t}\left(\frac{1}{2} \sum_{i, j=1}^{d} a_{i j} \partial_{i} \partial_{j} v+\sum_{i=1}^{d} g_{i} \partial_{i} v\right)\left(X_{s}\right) d s, \quad t \geq 0
$$


is a continuous square integrable local $\mathbb{P}_{x}$-martingale with respect to the stopping times $\left(D_{n}\right)_{n \geq 1}$ for any $x \in \mathbb{R}^{d}$. Indeed, let $\left(v_{n}\right)_{n \geq 1} \subset C_{0}^{2}\left(\mathbb{R}^{d}\right)$ be such that $v_{n}=v$ pointwise on $\bar{B}_{n}, n \geq 1$. Then for any $n \geq 1$, we have $\mathbb{P}_{x}$-a.s

$$
M_{t \wedge D_{n}}^{v}=M_{t \wedge D_{n}}^{v_{n}}, \quad t \geq 0,
$$

and $\left(M_{t \wedge D_{n}}^{v_{n}}\right)_{t \geq 0}$ is a square integrable $\mathbb{P}_{x}$-martingale for any $x \in \mathbb{R}^{d}$ by Proposition 3.17. Now let $u_{i} \in C^{2}\left(\mathbb{R}^{d}\right), i=1, \ldots, d$, be the coordinate projections, i.e. $u_{i}(x)=x_{i}$. Then by Proposition 3.17 polarization and localization with respect to $\left(D_{n}\right)_{n \geq 1}$, the quadratic covariation processes satisfy

$$
\left\langle M^{u_{i}}, M^{u_{j}}\right\rangle_{t}=\int_{0}^{t} a_{i j}\left(X_{s}\right) d s, \quad 1 \leq i, j \leq d, t \geq 0 .
$$

Using Lemma 3.18 we obtain by [20, II. Theorem 7.1] that there exists a $d$-dimensional Brownian motion $\left(W_{t}\right)_{t \geq 0}=\left(W_{t}^{1}, \ldots, W_{t}^{d}\right)_{t \geq 0}$ on $\left(\Omega, \mathcal{F},\left(\mathcal{F}_{t}\right)_{t \geq 0}, \mathbb{P}_{x}\right), x \in \mathbb{R}^{d}$, such that

$$
M_{t}^{u_{i}}=\sum_{j=1}^{d} \int_{0}^{t} \sigma_{i j}\left(X_{s}\right) d W_{s}^{j}, \quad 1 \leq i \leq d, t \geq 0 .
$$

Since for any $x \in \mathbb{R}^{d}, \mathbb{P}_{x^{-a . s . ~}}$

$$
M_{t}^{u_{i}}=X_{t}^{i}-x_{i}-\int_{0}^{t} g_{i}\left(X_{s}\right) d s, \quad t \geq 0
$$

the assertion follows.

(ii) The proof of (ii) is similar to the proof of (i) but uses [20, II. Theorem 7.1'] instead of [20, II. Theorem 7.1] (see [20, IV. Proposition 2.1])

Remark 3.20 Theorem 3.19 holds in general only up to $\zeta$, when one does not impose nonexplosion. Here, we only sketch in detail the proof in case of Theorem 3.19(i). (The case of Theorem 3.19(ii) is nearly the same but one has to work on a standard extension of the underlying probability space). One first uses that for $v_{k} \in C_{0}^{2}\left(\mathbb{R}^{d}\right), 1 \leq k \leq d$, one has by Proposition 3.17

$$
\left\langle M^{v_{k}}, M^{v_{l}}\right\rangle_{t}=\int_{0}^{t} \Phi_{k l}\left(X_{s}\right) d s, \quad 1 \leq k, l \leq d, t \geq 0
$$

where $\Phi_{k l}=\sum_{i, j=1}^{d} a_{i j} \partial_{j} v_{k} \partial_{i} v_{l}$, so that

$$
\Phi_{k l}=\sum_{m=1}^{d} \Psi_{k m} \Psi_{l m}, \quad \text { with } \quad \Psi_{k m}=\sum_{i=1}^{d} \sigma_{i m} \partial_{i} v_{k}, \quad 1 \leq k, l, m \leq d .
$$

Note that we then do no longer have

$$
\operatorname{det}\left(\left(\Psi_{k m}\right)_{1 \leq k, m \leq d}\right) \neq 0
$$

globally as opposed to Lemma 3.18. However, choosing $v_{k}(x)=v_{k}^{n}(x)=x_{k}$ on $\bar{B}_{n}, 1 \leq k \leq d$, $n \geq 1$, we can obtain (47) locally on $B_{n}$, hence (45) locally on $\left\{t \leq D_{n}\right\}$ for each $n \geq 1$. Consequently, we also get (46) locally on $\left\{t \leq D_{n}\right\}$ for each $n \geq 1$. Then showing consistency of the local martingale and drift parts, we obtain (44) up to $\zeta$ by Lemma 3.15(i). 


\section{Long time behavior, moment inequalities and uniqueness of invariant probability measures}

In this section we investigate long time behavior like non-explosion, recurrence and ergodicity. We will also investigate some moment inequalities that are well-known for classical Itô equations with continuous coefficients. We saw in Theorem 3.19 and Remark 3.20 that we can obtain a weak solution up to the life time $\zeta$. We first provide explicit non-explosion criteria, i.e. explicit criteria that imply the assumption

$$
\mathbb{P}_{x}(\zeta=\infty)=1 \text { for any } x \in \mathbb{R}^{d}
$$

of Theorem 3.19 .

\subsection{Non-explosion criteria and moment inequalities}

\subsubsection{Non-explosion criteria and moment inequalities without involving the density $\rho$}

In this subsection we consider non-explosion criteria that only depend on the coefficients of the underlying SDE. We first derive a lemma that is a variant of the construction in [7, page 197] and then a non-explosion criterion by following a probabilistic technique which traces back at least to [31, 10.2].

Lemma 4.1 Let $f \in C^{2}\left(\mathbb{R}^{d}\right)$ be a positive, strictly increasing and unbounded radial function, i.e. $f \geq 0$ pointwise, $f(x) \equiv c_{r}$ on $\partial B_{r}$ with $0<c_{r}<c_{r^{\prime}}$ whenever $0<r<r^{\prime}$, and $c_{n} \rightarrow \infty$ as $n \rightarrow \infty$. Under the assumptions on the coefficients of Theorem 3.2, suppose that there exist $M>0, N_{0} \in \mathbb{N}$ such that

$$
L f=\frac{1}{2} \sum_{i, j=1}^{d} a_{i j} \partial_{i} \partial_{j} f+\sum_{i=1}^{d} g_{i} \partial_{i} f \leq M f \quad \text { a.e. on } \mathbb{R}^{d} \backslash B_{N_{0}} .
$$

Let $\phi \in C^{2}(\mathbb{R})$, such that $\phi, \phi^{\prime} \geq 0$ pointwise,

$$
\phi(t)= \begin{cases}\sup _{B_{N_{0}}} f & \text { if } t \leq \sup _{B_{N_{0}}} f \\ t & \text { if } t \geq \sup _{B_{N_{0}+1}} f\end{cases}
$$

and let for arbitrary $\alpha \geq 0$

$$
\psi:=\phi \circ f+C_{\phi, A}+\alpha,
$$

where

$$
C_{\phi, A}:=M\left(c_{\phi} \sup _{B_{N_{0}+1}} f+\frac{c_{\phi}}{2 M} \sup _{B_{N_{0}+1}}\langle A \nabla f, \nabla f\rangle\right)
$$

and

$$
c_{\phi}:=\sup _{B_{N_{0}+1} \backslash \bar{B}_{N_{0}}} \phi^{\prime} \circ f+\sup _{B_{N_{0}+1} \backslash \bar{B}_{N_{0}}}\left|\phi^{\prime \prime} \circ f\right| .
$$

Then $\psi \in C^{2}\left(\mathbb{R}^{d}\right), \psi>0$ pointwise, $\inf _{\partial B_{n}} \psi \nearrow \nearrow$ as $n \rightarrow \infty, n \geq N_{0}$, and

$$
L \psi \leq M \psi \quad \text { a.e. on } \mathbb{R}^{d} .
$$


Proof Using the formula

$$
L(\phi(f))=\phi^{\prime}(f) L f+\frac{1}{2} \phi^{\prime \prime}(f)\langle A \nabla f, \nabla f\rangle
$$

the assertion is easily verified.

Theorem 4.2 Suppose that (3) holds. Then for $\mathbb{M}$ as in Theorem 3.12 it holds

$$
\mathbb{P}_{x}(\zeta=\infty)=1 \text { for any } x \in \mathbb{R}^{d} .
$$

Proof We first show the statement corresponding to (3). Let $u_{n} \in C_{0}^{2}\left(\mathbb{R}^{d}\right), n \geq 1$, be positive functions such that

$$
u_{n}(x)= \begin{cases}\|x\|^{2} & \text { if } x \in \bar{B}_{n}, \\ 0 & \text { if } x \in \mathbb{R}^{d} \backslash B_{n+1} .\end{cases}
$$

Then by Proposition 3.16

$$
Y_{t}^{n}:=u_{n}\left(X_{t}\right), t \geq 0
$$

is a positive continuous $\mathbb{P}_{x}$-semimartingale for any $x \in \mathbb{R}^{d}, n \geq 1$.

Let $f(x)=\ln \left(\|x\|^{2}+1\right)+1, x \in \mathbb{R}^{d}$ and let $\psi, \phi$ and $C_{\phi, A}$ be as in Lemma 4.1 with $\alpha=0$. By Itô's formula applied to $Y^{n}$ with the function $e^{-M t} \varphi(y)$,

$$
\varphi(y):=\phi(\ln (1+y)+1)+C_{\phi, A},
$$

we obtain $\mathbb{P}_{x}$-a.s. for any $x \in B_{n}$

$$
e^{-M t} \varphi\left(Y_{t}^{n}\right)=\varphi\left(Y_{0}^{n}\right)+\int_{0}^{t} e^{-M s} \varphi^{\prime}\left(Y_{s}^{n}\right) d M_{s}^{u_{n}}+\int_{0}^{t} e^{-M s}(L-M)\left(\varphi \circ u_{n}\right)\left(X_{s}\right) d s .
$$

Note that $(L-M)\left(\varphi \circ u_{n}\right)=(L-M) \psi \leq 0 m$-a.e. on $\bar{B}_{n}$ for each $n \geq 1$. Therefore, using the last part of Lemma 3.14(i), we can see that

$$
e^{-M t \wedge \sigma_{n}} \varphi \circ u_{n}\left(X_{t \wedge \sigma_{n}}\right), t \geq 0
$$

is a positive continuous $\mathbb{P}_{x}$-supermartingale for any $x \in B_{n}, n \geq 1$. Since $\mathbb{M}$ has continuous sample paths on the one-point-compactification $\mathbb{R}_{\Delta}^{d}$, we have that $\left\|X_{t \wedge \sigma_{n}}\right\|=n \mathbb{P}_{x^{-}}$-a.s. on $\left\{\sigma_{n} \leq t\right\}$ for any $x \in B_{n}$. Now let $x \in \mathbb{R}^{d}$ be arbitrary. Then $x \in B_{k_{0}}$ for some $k_{0} \in \mathbb{N}$ and since supermartingales have decreasing expectations, we get for any $n>k_{0}$

$$
\begin{aligned}
\phi\left(\ln \left(\|x\|^{2}+1\right)+1\right)+C_{\phi, A} & =\mathbb{E}_{x}\left[\varphi \circ u_{n}\left(X_{0}\right)\right] \\
& \geq \mathbb{E}_{x}\left[e^{-M t \wedge \sigma_{n}} \varphi \circ u_{n}\left(X_{t \wedge \sigma_{n}}\right)\right] \\
& \geq e^{-M t} \mathbb{E}_{x}\left[\varphi \circ u_{n}\left(X_{t \wedge \sigma_{n}}\right) 1_{\left\{\sigma_{n} \leq t\right\}}\right] \\
& \geq e^{-M t}\left(\phi\left(\ln \left(n^{2}+1\right)+1\right)+C_{\phi, A}\right) \mathbb{P}_{x}\left(\sigma_{n} \leq t\right) .
\end{aligned}
$$

Consequently

$$
\mathbb{P}_{x}(\zeta \leq t)=\lim _{n \rightarrow \infty} \mathbb{P}_{x}\left(\sigma_{n} \leq t\right)=0
$$

for any $t \geq 0$, which implies the assertion. 
Remark 4.3 (i) Suppose that for the semigroup $\left(T_{t}\right)_{t>0}$ defined on $L^{\infty}\left(\mathbb{R}^{d}, m\right)$ it holds

$$
T_{t} 1_{\mathbb{R}^{d}}=1 \text { m-a.e. for some (and hence all) } t>0 \text {. }
$$

Then, since $T_{t} 1_{\mathbb{R}^{d}}=P_{t} 1_{\mathbb{R}^{d}} m$-a.e. and $P_{t} 1_{\mathbb{R}^{d}}$ is continuous by the strong Feller property (cf. Proposition $3.10($ ii) )

$$
P_{t} 1_{\mathbb{R}^{d}}(x)=1 \text { for any } x \in \mathbb{R}^{d}, t>0 \text {, or equivalently } \mathbb{M} \text { is non-explosive. }
$$

(ii) Using (i), the non-explosion criterion (3) can be recovered form the dual version of [30, Proposition 1.10]. Indeed, (48) holds, if and only if $m$ is invariant for the $L^{1}\left(\mathbb{R}^{d}, m\right)$-semigroup $\left(\widehat{T}_{t}\right)_{t>0}$. Then Theorem 4.2 follows by applying the dual version of [30, Proposition 1.10(b)] to the $C^{2}$-function $\psi$ as defined in the proof of Theorem 4.2 and then using (49).

(iii) In general, $\mathbb{M}$ will be non-explosive whenever there exists $\psi \in C^{2}\left(\mathbb{R}^{d}\right)$ and $M>0$, such that $\inf _{\partial B_{n}} \psi \rightarrow \infty$ as $n \rightarrow \infty$ and $L \psi \leq M \psi$ a.e. on $\mathbb{R}^{d}$. This follows from [30, Proposition 1.10] and (i), and can be shown as well by applying the technique of supermartingales from Theorem [4.2, using a generalized version of Lemma [4.1 (see [7, page 197]), and noting that $\left(M_{t \wedge D_{n}}^{v}\right)_{t \geq 0}$, is a martingale for any $v \in C^{2}\left(\mathbb{R}^{d}\right)$ (see proof of Theorem [3.19(i)). Note the subtle difference that [30, Proposition 1.10] is proved by analytic means (starting from the $L^{1}$-generator or $L^{1}$-semigroup) and only leads to (48), whereas Theorem 4.2 is proven by probabilistic means (starting from Proposition 3.16) and directly leads to (49) regardless of the classical strong Feller property.

Theorem 4.4 (i) Assume for some $N_{0} \in \mathbb{N}$ and some $p>0$, there exists $M>0$ such that

$$
\left(\frac{p-2}{2}\right) \frac{\langle A(x) x, x\rangle}{\|x\|^{2}+1}+\frac{1}{2} \operatorname{trace} A(x)+\langle\mathbf{G}(x), x\rangle \leq M\left(\|x\|^{2}+1\right),
$$

for a.e. $x \in \mathbb{R}^{d} \backslash B_{N_{0}}$. Then $\mathbb{M}$ of Theorem 3.12 is non-explosive and for any open ball $B$ there exists a constant $C_{B}>0$, such that

$$
\sup _{x \in \bar{B}} \mathbb{E}_{x}\left[\left\|X_{t}\right\|^{p}\right] \leq C_{B} \cdot e^{M \cdot t}, \quad \forall t \geq 0 .
$$

(ii) Let $\sigma=\left(\sigma_{i j}\right)_{1 \leq i, j \leq d}$ be as in Lemma 3.18 and and $\mathbf{G}$ as in Theorem 3.2. Assume that for some $N_{0} \in \mathbb{N}$ and $C_{1}>0$

$$
\max _{1 \leq i, j \leq d}\left|\sigma_{i j}(x)\right|+\max _{1 \leq i \leq d}\left|g_{i}(x)\right| \leq C_{1}(\|x\|+1) \quad \text { for a.e. } x \in \mathbb{R}^{d} \backslash B_{N_{0}} .
$$

Then $\mathbb{M}$ of Theorem 3.12 is non-explosive and for any $T>0$, and any open ball $B$, there exist constants $C_{T, B}, C_{T}$ such that

$$
\sup _{x \in \bar{B}} \mathbb{E}_{x}\left[\sup _{s \leq t}\left\|X_{s}\right\|^{2}\right] \leq C_{T, B} \cdot e^{C_{T} \cdot t}, \quad \forall t \leq T .
$$

Proof (i) Let $f(x)=\left(\|x\|^{2}+1\right)^{\frac{p}{2}}$. Then (50) implies $L f(x) \leq M p \cdot f(x)$ for a.e. $x \in \mathbb{R}^{d} \backslash B_{N_{0}}$. Let $\phi, \psi$, and $C_{\phi, A}$ be as in Lemma 4.1 with $\alpha:=\sup _{B_{N_{0}+1}} f$.

Let $\varphi(y):=\phi\left((y+1)^{\frac{p}{2}}\right)+C_{\phi, A}+\alpha$. Applying Itô's formula to $u_{n}(X$.$) , where u_{n}$ is as in the proof 
of Theorem 4.2, with the function $e^{-M p \cdot t} \varphi(y)$, we obtain exactly as in the proof of Theorem 4.2 that $\mathbb{M}$ is non-explosive. For arbitrary $n \in \mathbb{N}$ and $x \in B_{n}$ it holds

$$
\left(C_{\phi, A}+2 \sup _{B_{N_{0}+1}} f\right) f(x) \geq \psi(x) \geq \mathbb{E}_{x}\left[e^{-(M \cdot p) t \wedge \sigma_{n}} \varphi \circ u_{n}\left(X_{t \wedge \sigma_{n}}\right)\right] .
$$

Using $f \leq \psi$ pointwise, $\sigma_{n} \nearrow \infty$, Fatou's lemma and the previous inequality, we get

$$
e^{-M p \cdot t} \mathbb{E}_{x}\left[f\left(X_{t}\right)\right] \leq \liminf _{n \rightarrow \infty} \mathbb{E}_{x}\left[e^{-(M \cdot p) t \wedge \sigma_{n}} \varphi \circ u_{n}\left(X_{t \wedge \sigma_{n}}\right)\right] \leq\left(C_{\phi, A}+2 \sup _{B_{N_{0}}+1} f\right) f(x) .
$$

Thus,

$$
\mathbb{E}_{x}\left[\left\|X_{t}\right\|^{p}\right] \leq \underbrace{\left(C_{\phi, A}+2 \sup _{B_{N_{0}+1}} f\right)\left(\|x\|^{2}+1\right)^{\frac{p}{2}}}_{=: C_{x}} e^{M p \cdot t} .
$$

Now set $C_{B}:=\sup _{x \in \bar{B}} C_{x}$.

(ii) (51) implies

$$
\operatorname{trace}(A(x))=\sum_{i, j=1}^{d} \sigma_{i j}(x)^{2} \leq 2 d^{2} C_{1}^{2}\left(\|x\|^{2}+1\right) \quad \text { for a.e. } x \in \mathbb{R}^{d} \backslash B_{N_{0}}
$$

and

$$
\langle\mathbf{G}(x), x\rangle \leq\left(\sum_{i=1}^{d} g_{i}(x)^{2}\right)^{\frac{1}{2}}\|x\| \leq 2 d C_{1}\left(\|x\|^{2}+1\right) \quad \text { for a.e. } x \in \mathbb{R}^{d} \backslash B_{N_{0}} .
$$

Thus (3) holds, so that $\mathbb{M}$ is non-explosive by Theorem 4.2 and (44) holds. Consequently, $\mathbb{P}_{x}$-a.s. for any $1 \leq i \leq d$

$$
\begin{aligned}
& \sup _{0 \leq s \leq t \wedge \sigma_{n}}\left|X_{s}^{i}\right|^{2} \\
\leq & (d+2)\left(x_{i}^{2}+\sum_{j=1}^{d} \sup _{0 \leq s \leq t \wedge \sigma_{n}}\left|\int_{0}^{s} \sigma_{i j}\left(X_{u}\right) d W_{u}^{j}\right|^{2}+t \int_{0}^{t \wedge \sigma_{n}}\left|g_{i}\left(X_{u}\right)\right|^{2} d u\right) .
\end{aligned}
$$

Note that $\sum_{i, j=1}^{d} \sigma_{i j}(x)^{2}=\operatorname{trace}(A(x)) \leq d \cdot \Lambda_{B_{N_{0}}} \leq d \cdot \Lambda_{B_{N_{0}}}\left(\|x\|^{2}+1\right)$ for a.e. $x \in B_{N_{0}}$. Thus

$$
\begin{aligned}
& \sum_{i, j=1}^{d} \mathbb{E}_{x}\left[\sup _{0 \leq s \leq t \wedge \sigma_{n}}\left|\int_{0}^{s} \sigma_{i j}\left(X_{u}\right) d W_{u}^{j}\right|^{2}\right] \\
& \leq 4 \mathbb{E}_{x}\left[\sum_{i, j=1}^{d}\left\langle\int_{0}^{.} \sigma_{i j}\left(X_{u}\right) d W_{u}^{j}\right\rangle_{t \wedge \sigma_{n}}\right] \\
& \leq 4 \mathbb{E}_{x}\left[\sum_{i, j=1}^{d} \int_{0}^{t \wedge \sigma_{n}} \sigma_{i j}^{2}\left(X_{u}\right) d u\right] \\
& \leq 4\left(2 d^{2} C_{1}^{2}+d \Lambda_{B_{N_{0}}}\right) \\
& \mathbb{E}_{x}\left[\int_{0}^{t \wedge \sigma_{n}}\left(\left\|X_{u}\right\|^{2}+1\right) d u\right] \\
& \leq C_{2} \int_{0}^{t} \mathbb{E}_{x}\left[\sup _{0 \leq s \leq u \wedge \sigma_{n}}\left\|X_{s}\right\|^{2}\right] d u+C_{2} T .
\end{aligned}
$$


Now let $x \in \bar{B}$, and $\forall t \leq T$. Then using (40), (51), for any $n \in \mathbb{N}$ and $1 \leq i \leq d$

$$
\begin{aligned}
& \mathbb{E}_{x}\left[\int_{0}^{t \wedge \sigma_{n}}\left|g_{i}\left(X_{u}\right)\right|^{2} d u\right] \\
& \leq \mathbb{E}_{x}\left[\int_{0}^{T}\left|g_{i} 1_{B_{N_{0}}}\right|^{2}\left(X_{u}\right) d u\right]+\mathbb{E}_{x}\left[\int_{0}^{t \wedge \sigma_{n}}\left|g_{i} 1_{\mathbb{R}^{d} \backslash B_{N_{0}}}\right|^{2}\left(X_{u}\right) d u\right] \\
& \leq c_{B, p} e^{T}\left\|g_{i} 1_{B_{N_{0}}}\right\|_{L^{p}\left(\mathbb{R}^{d}, m\right)}^{2}+2 C_{1} \mathbb{E}_{x}\left[\int_{0}^{t \wedge \sigma_{n}}\left(\left\|X_{u}\right\|^{2}+1\right) d u\right] \\
& \leq c_{B, p} e^{T} \sup _{\bar{B}_{N_{0}}}|\rho|^{\frac{2}{p}} \cdot\left\|g_{i}\right\|_{L^{p}\left(B_{N_{0}}\right)}^{2}+2 C_{1} \int_{0}^{t} \mathbb{E}_{x}\left[\sup _{0 \leq s \leq u \wedge \sigma_{n}}\left\|X_{s}\right\|^{2}\right] d u+2 C_{1} T .
\end{aligned}
$$

Now let $h_{n}(t):=\mathbb{E}_{x}\left[\sup _{0 \leq u \leq t \wedge \sigma_{n}}\left\|X_{u}\right\|^{2}\right]$. Then by (52), (53), (54), we obtain

$$
\begin{aligned}
h_{n}(t) \leq & \underbrace{(d+2)\|x\|^{2}+C_{2} T+c_{B, p} e^{T} T \sup _{\bar{B}_{N_{0}}}|\rho|^{\frac{2}{p}} \cdot\left(\sum_{i=1}^{d}\left\|g_{i}\right\|_{L^{p}\left(B_{N_{0}}\right)}^{2}\right)+2 d C_{1} T^{2}}_{=: C_{T, B}} \\
& +\underbrace{\left(2 d C_{1} T+C_{2}\right.}_{=: C_{T}}) \int_{0}^{t} h_{n}(u) d u .
\end{aligned}
$$

By Gronwall's inequality, $h_{n}(t) \leq C_{T, B} \cdot e^{C_{T} \cdot t}$. Since none of the involved constants depends on $n$, we can use Fatou's lemma letting $n \rightarrow \infty$, and obtain

$$
\mathbb{E}_{x}\left[\sup _{s \leq t}\left\|X_{s}\right\|^{2}\right] \leq C_{T, B} e^{C_{T} \cdot t}, \quad \forall t \leq T .
$$

Since $x \in \bar{B}$ was arbitrary, the desired result follows.

\subsubsection{Non-explosion criteria involving the density $\rho$}

By [30, Proposition 1.10] (a) we know that (48) holds, whenever

$$
a_{i j}, g_{i}-\beta_{i}^{\rho, A} \in L^{1}\left(\mathbb{R}^{d}, m\right), \quad 1 \leq i, j \leq d .
$$

Thus (55) provides a sufficient condition for non-explosion of $\mathbb{M}$ as in Theorem 3.12 by (49) which obviously depends on the knowledge of the density $\rho$.

A systematic study of non-explosion conditions, more precisely results implying (48) and involving the density $\rho$ can be found in [16, Corollary 15].

\subsection{Recurrence criteria and other ergodic properties involving and not involving the density $\rho$}

The measure $m=\rho d x$, where the density $\rho$ is as at the beginning of Section 3 or as in Theorem 3.2. can be seen to define a stationary distribution. In fact, if the $L^{1}\left(\mathbb{R}^{d}, m\right)$-semigroup $\left(\widehat{T}_{t}\right)_{t>0}$ 
is conservative, for instance if (55) holds or there exists a constant $M \geq 0$ and some $N_{0} \in \mathbb{N}$, such that

$$
-\frac{\langle A(x) x, x\rangle}{\|x\|^{2}+1}+\frac{1}{2} \operatorname{trace}(A(x))+\left\langle\left(2 \beta^{\rho, A}-\mathbf{G}\right)(x), x\right\rangle \leq M\left(\|x\|^{2}+1\right)\left(\ln \left(\|x\|^{2}+1\right)+1\right)
$$

for a.e. $x \in \mathbb{R}^{d} \backslash B_{N_{0}}$, as one can see from the dual version of Theorem 4.2 or [30, Proposition $1.10(\mathrm{c})]$, then $m$ is an invariant measure (for $\left.\left(T_{t}\right)_{t>0}\right)$, i.e. for any $f \in L^{1}\left(\mathbb{R}^{d}, m\right)$

$$
\int_{\mathbb{R}^{d}} T_{t} f d m=\int_{\mathbb{R}^{d}} f \widehat{T}_{t} 1_{\mathbb{R}^{d}} d m=\int_{\mathbb{R}^{d}} f d m
$$

so that for any $A \in \mathcal{B}\left(\mathbb{R}^{d}\right)$ and $t \geq 0$

$$
\begin{aligned}
\mathbb{P}_{m}\left(X_{t} \in A\right) & :=\int_{\mathbb{R}^{d}} \mathbb{P}_{x}\left(X_{t} \in A\right) m(d x)=\int_{\mathbb{R}^{d}} T_{t} 1_{A}(x) m(d x) \\
& =\lim _{n \rightarrow \infty} \int_{\mathbb{R}^{d}} T_{t} 1_{A \cap B_{n}}(x) m(d x)=\lim _{n \rightarrow \infty} \int_{\mathbb{R}^{d}} 1_{A \cap B_{n}}(x) m(d x)=m(A) .
\end{aligned}
$$

However, usually $m$ is not a probability measure, hence $\mathbb{P}_{m}$ is also not such a measure. But if it is, then $\mathbb{P}_{m}$ is a stationary distribution (if $\left(\widehat{T}_{t}\right)_{t>0}$ is conservative). Main parts of the monograph [7] focus on the density $\rho$ or more generally on $m$, in case $m$ is a probability measure and aim in deriving properties of both (since both are in general not explicit).

We will first consider possibly infinite $m$ and we may assume that $\rho$ is explicit as is explained in the following remark.

Remark 4.5 All results up to now and further hold exactly in the same form, if we assume that $\rho \in C_{\text {loc }}^{0,1-d / p}\left(\mathbb{R}^{d}\right) \cap H_{\text {loc }}^{1, p}\left(\mathbb{R}^{d}\right)$ for some $p>d$ with $\rho(x)>0$ for all $x \in \mathbb{R}^{d}$ is explicitly given from the beginning, that $A:=\left(a_{i j}\right)_{1 \leq i, j \leq d}$ is as in Theorem 3.2 and that $\mathbf{B}=\left(b_{1}, \ldots, b_{d}\right) \in$ $L_{l o c}^{p}\left(\mathbb{R}^{d}, \mathbb{R}^{d}\right)$ satisfies

$$
\int_{\mathbb{R}^{d}}\langle\mathbf{B}, \nabla f\rangle d m=0, \quad \forall f \in C_{0}^{\infty}\left(\mathbb{R}^{d}\right)
$$

Indeed, we then just have to set $\mathbf{G}:=\beta^{\rho, A}+\mathbf{B}$. Then all conclusions of Theorem 3.2 hold with the explicitly chosen density from above. Note that this also includes the setting of Theorem 3.2 since by its conclusion a $\rho$ like above exists and can hence be "explicitly" chosen.

We want to derive explicit conditions for recurrence involving and not involving the density $\rho$ in two general cases where $m$ is a general $\sigma$-finite measure and where $m$ is a finite, yet without loss of generality a probability measure. First, we derive a lemma which leads to irreducibility in the probabilistic sense and strict irreducibility in the sense of generalized Dirichlet forms (see Corollary 4.8) and as a byproduct leads to a weaker condition for non-explosion (see Remark 4.7).

Lemma 4.6 Under the assumptions of Remark 4.5, it holds:

(i) Let $A \in \mathcal{B}\left(\mathbb{R}^{d}\right)$ be such that $P_{t_{0}} 1_{A}\left(x_{0}\right)=0$ for some $t_{0}>0$ and $x_{0} \in \mathbb{R}^{d}$. Then $m(A)=0$.

(ii) Let $A \in \mathcal{B}\left(\mathbb{R}^{d}\right)$ be such that $P_{t_{0}} 1_{A}\left(x_{0}\right)=1$ for some $t_{0}>0$ and $x_{0} \in \mathbb{R}^{d}$. Then $P_{t} 1_{A}(x)=1$ for all $(x, t) \in \mathbb{R}^{d} \times(0, \infty)$. 
Proof (i) Suppose $m(A)>0$. Choose an open ball $B_{r}\left(x_{0}\right) \subset \mathbb{R}^{d}$ such that

$$
0<m\left(A \cap B_{r}\left(x_{0}\right)\right)<\infty .
$$

Let $u:=\rho P .1_{A \cap B_{r}\left(x_{0}\right)}$. Then $0=u\left(x_{0}, t_{0}\right) \leq \rho\left(x_{0}\right) P_{t_{0}} 1_{A}\left(x_{0}\right)=0$. Take $f_{n} \in C_{0}^{\infty}\left(\mathbb{R}^{d}\right)$ with $f_{n} \geq 0$ such that $f_{n} \rightarrow 1_{A \cap B_{r}\left(x_{0}\right)}$ in $L^{1}\left(\mathbb{R}^{d}, m\right)$. Then by (30) and the explanation right after it, for arbitrary bounded open set $U$ in $\mathbb{R}^{d}$ and $\left[\tau_{1}, \tau_{2}\right] \subset(0, \infty)$, there is some $\gamma \in(0,1)$ such that

$$
P . f_{n}(\cdot) \rightarrow P .1_{A \cap B_{r}\left(x_{0}\right)}(\cdot) \text { in } C^{\gamma ; \frac{\gamma}{2}}\left(\bar{U} \times\left[\tau_{1}, \tau_{2}\right]\right),
$$

hence

$$
u_{n}:=\rho P . f_{n} \rightarrow u \text { in } C^{\gamma ; \frac{\gamma}{2}}\left(\bar{U} \times\left[\tau_{1}, \tau_{2}\right]\right) .
$$

Fix $T>t_{0}$ and $U \supset \bar{B}_{r}\left(x_{0}\right)$. Then (see proof of Theorem 3.8) for all $\varphi \in C_{0}^{\infty}(U \times(0, T)$ )

$$
\int_{0}^{T} \int_{U}\left(\frac{1}{2}\left\langle A \nabla u_{n}, \nabla \varphi\right\rangle+u_{n}\langle\beta, \nabla \varphi\rangle-u_{n} \partial_{t} \varphi\right) d x d t=0
$$

where $\beta$ is defined as in the proof of Theorem 3.8. Now take arbitrary but fixed $(x, t) \in B_{r}\left(x_{0}\right) \times$ $\left(0, t_{0}\right)$. By [2, Theorem 5]

$$
0 \leq u_{n}(x, t) \leq u_{n}\left(x_{0}, t_{0}\right) \exp \left(C\left(\frac{\left\|x_{0}-x\right\|^{2}}{t_{0}-t}+\frac{t_{0}-t}{\min (1, t)}+1\right)\right)
$$

and (57) applied with $U \supset \bar{B}_{r}\left(x_{0}\right),\left[\tau_{1}, \tau_{2}\right] \supset\left[t, t_{0}\right]$ then leads to

$$
0 \leq u(x, t) \leq u\left(x_{0}, t_{0}\right) \exp \left(C\left(\frac{\left\|x_{0}-x\right\|^{2}}{t_{0}-t}+\frac{t_{0}-t}{\min (1, t)}+1\right)\right)=0 .
$$

Thus, $P_{t} 1_{A \cap B_{r}\left(x_{0}\right)}(x)=0$ for all $x \in B_{r}\left(x_{0}\right)$ and $0<t<t_{0}$, so that

$$
0=\int_{\mathbb{R}^{d}} 1_{A \cap B_{r}\left(x_{0}\right)} P_{t} 1_{A \cap B_{r}\left(x_{0}\right)} d m \underset{\text { as } \underset{t \rightarrow 0}{\longrightarrow}}{\longrightarrow} m\left(B_{r}\left(x_{0}\right) \cap A\right)>0,
$$

which is contradiction. Therefore, we must have $m(A)=0$.

(ii) Let $y \in \mathbb{R}^{d}$ and $0<s<t_{0}$ be arbitrary but fixed and let $r:=2\left\|x_{0}-y\right\|$ and let $B$ be any open ball. Take $g_{n} \in C_{0}^{\infty}\left(\mathbb{R}^{d}\right)$ with $0 \leq g_{n} \leq 1$ such that $g_{n} \rightarrow 1_{A \cap B}$ in $L^{1}\left(\mathbb{R}^{d}, m\right)$. Then by (30) and the explanation right after it, there is some $\gamma \in(0,1)$ such that

$$
P . g_{n}(\cdot) \longrightarrow P .1_{A \cap B}(\cdot) \text { in } C^{\gamma ; \frac{\gamma}{2}}\left(\bar{B}_{r}\left(x_{0}\right) \times\left[s / 2,2 t_{0}\right]\right) .
$$

Fix $T>0$ and $U \supset \bar{B}_{r}\left(x_{0}\right)$. Using the property

$$
\beta=\frac{1}{2} \nabla A+\mathbf{G}-2 \beta^{\rho, A}=\mathbf{B}-\beta^{\rho, A}+\frac{1}{2} \nabla A=\mathbf{B}-\frac{1}{2} A \frac{\nabla \rho}{\rho},
$$

and (66), we directly get for all $\varphi \in C_{0}^{\infty}(U \times(0, T))$

$$
\int_{0}^{T} \int_{U}\left(\frac{1}{2}\langle A \nabla \rho, \nabla \varphi\rangle+\rho\langle\beta, \nabla \varphi\rangle-\rho \partial_{t} \varphi\right) d x d t=\int_{0}^{T}\left(\int_{U}\langle\mathbf{B}, \nabla \varphi\rangle \rho d x\right) d t=0
$$


and (cf. the proof of Theorem 3.8) we also get

$$
\int_{0}^{T} \int_{U}\left(\frac{1}{2}\left\langle A \nabla\left(\rho P . g_{n}\right), \nabla \varphi\right\rangle+\left(\rho P . g_{n}\right)\langle\beta, \nabla \varphi\rangle-\left(\rho P . g_{n}\right) \partial_{t} \varphi\right) d x d t=0 .
$$

Now let $u_{n}(x, t):=\rho(x)\left(1-P_{t} g_{n}(x)\right)$. Then $u_{n} \in H^{1,2}(U \times(0, T))$ and $u_{n} \geq 0$. Subtracting (61) from (60) implies

$$
\int_{0}^{T} \int_{U}\left(\frac{1}{2}\left\langle A \nabla u_{n}, \nabla \varphi\right\rangle+u_{n}\langle\beta, \nabla \varphi\rangle-u_{n} \partial_{t} \varphi\right) d x d t=0 .
$$

Thus, by [2, Theorem 5]

$$
0 \leq u_{n}(y, s) \leq u_{n}\left(x_{0}, t_{0}\right) \underbrace{\exp \left(C\left(\frac{\left\|x_{0}-y\right\|^{2}}{t_{0}-s}+\frac{t_{0}-s}{\min (1, s)}+1\right)\right)}_{=: C_{2}} .
$$

By (59)

$$
0 \leq \rho(y)\left(1-P_{s} 1_{A \cap B}(y)\right) \leq C_{2} \rho\left(x_{0}\right)\left(1-P_{t_{0}} 1_{A \cap B}\left(x_{0}\right)\right) .
$$

Note that for all $(x, t) \in \mathbb{R}^{d} \times(0, \infty), P_{t} 1_{A \cap B_{n}} 1(x) \nearrow P_{t} 1_{A}(x)$ as $n \rightarrow \infty$. Thus,

$$
0 \leq \rho(y)\left(1-P_{s} 1_{A}(y)\right) \leq C_{2} \rho\left(x_{0}\right)\left(1-P_{t_{0}} 1_{A}\left(x_{0}\right)\right)=0 .
$$

Consequently, $P_{s} 1_{A}(y)=1$ for any $(y, s) \in \mathbb{R}^{d} \times\left(0, t_{0}\right)$ which can be extended to $\mathbb{R}^{d} \times\left(0, t_{0}\right]$ by continuity. And by the sub-Markovian property, $P_{t_{0}} 1_{\mathbb{R}^{d}}(y)=1$ for any $y \in \mathbb{R}^{d}$. Now let $t \in(0, \infty)$ be given. Then there exists $k \in \mathbb{N} \cup\{0\}$ such that

$$
k t_{0}<t \leq(k+1) t_{0}
$$

and so $P_{t} 1_{A}=P_{k t_{0}+\left(t-k t_{0}\right)} 1_{A}=\underbrace{P_{t_{0}} \circ \cdots \circ P_{t_{0}}}_{k \text {-times }} \circ P_{t-k t_{0}} 1_{A}=1$.

Remark 4.7 Under the assumptions of Remark 4.5, by Lemma 4.6 (ii) we know that $\mathbb{M}$ is nonexplosive, if $\mathbb{P}_{x}(\zeta=\infty)=1$ for some $x \in \mathbb{R}^{d}$. More precisely, if $\mathbb{P}_{x_{0}}\left(X_{t_{0}} \in \mathbb{R}^{d}\right)=1$ for some $\left(x_{0}, t_{0}\right) \in \mathbb{R}^{d} \times(0, \infty)$, then $\mathbb{M}$ is non-explosive. This (together with Proposition $[3.10$, Lemma 3.14) generalizes and improves [4, Lemma 2.5] to possibly locally unbounded drift coefficient using a completely different and genuine proof.

$A \in \mathcal{B}\left(\mathbb{R}^{d}\right)$ is called weakly invariant relative to $\left(T_{t}\right)_{t>0}$, if

$$
T_{t}\left(f \cdot 1_{A}\right)(x)=0, \text { for } m \text {-a.e. } x \in \mathbb{R}^{d} \backslash A,
$$

for any $t>0, f \in L^{2}\left(\mathbb{R}^{d}, m\right)$. $\left(T_{t}\right)_{t>0}$ is said to be strictly irreducible, if for any weakly invariant set $A$ relative to $\left(T_{t}\right)_{t>0}$, we have $m(A)=0$ or $m\left(\mathbb{R}^{d} \backslash A\right)=0$.

Corollary 4.8 Under the assumptions of Remark 4.5, it holds:

(i) $\left(T_{t}\right)_{t>0}$ is strictly irreducible. 
(ii) Let $A \in \mathcal{B}\left(\mathbb{R}^{d}\right)$ with $m(A)>0$. Then $\mathbb{P}_{x}\left(X_{t} \in A\right)>0$ for all $x \in \mathbb{R}^{d}, t>0$, i.e. $\mathbb{M}$ is irreducible in the probabilistic sense.

Proof (i) Let $A \in \mathcal{B}\left(\mathbb{R}^{d}\right)$ be a weakly invariant set with $m\left(\mathbb{R}^{d} \backslash A\right) \neq 0$. Then by monotone approximation with the $L^{2}$-functions $1_{B_{n}}, n \geq 1$, we get for any $t>0 P_{t} 1_{A}(x)=0$, for $m$-a.e. $x \in \mathbb{R}^{d} \backslash A$. Then there exists $t_{0}>0$ and $x_{0} \in \mathbb{R}^{d} \backslash A$ such that $P_{t_{0}} 1_{A}\left(x_{0}\right)=0$. By Lemma 4.6(i), we have $m(A)=0$, as desired.

(ii) By contraposition of Lemma 4.6(i), $\mathbb{P}_{x}\left(X_{t} \in A\right)=P_{t} 1_{A}(x)>0$, for all $x \in \mathbb{R}^{d}, t>0$.

\subsubsection{Explicit recurrence criteria for possibly infinite $m$}

We continue with some further definitions. Define the last exit time $L_{A}$ from $A \in \mathcal{B}\left(\mathbb{R}^{d}\right)$ by

$$
L_{A}:=\sup \left\{t \geq 0: X_{t} \in A\right\}, \quad(\sup \emptyset:=0) .
$$

$\mathbb{M}$ is called recurrent (in the probabilistic sense), if for any $\emptyset \neq U \subset \mathbb{R}^{d}, U$ open, we have

$$
\mathbb{P}_{x}\left(L_{U}=\infty\right)=1, \quad \forall x \in \mathbb{R}^{d} .
$$

Let $\left(\vartheta_{t}\right)_{t \geq 0}$ be the shift operator of $\mathbb{M}$. Using the shift invariance of $\Lambda:=\left\{L_{U}=\infty\right\}$, the Markov property and the strong Feller property of $\left(P_{t}\right)_{t>0}$, we get for all $x \in \mathbb{R}^{d}, t>0$

$$
\mathbb{P}_{x}(\Lambda)=\mathbb{P}_{x}\left(\vartheta_{t}^{-1}(\Lambda)\right)=\mathbb{E}_{x}\left[\mathbb{E}_{x}\left[1_{\Lambda} \circ \vartheta_{t} \mid \mathcal{F}_{t}\right]\right]=\mathbb{E}_{x}\left[\mathbb{E}_{X_{t}}\left[1_{\Lambda}\right]\right]=P_{t} \mathbb{E} \cdot\left[1_{\Lambda}\right](x)
$$

Thus

$$
\text { (62) } \Longleftrightarrow \mathbb{P}_{x}\left(L_{U}=\infty\right)=1 \quad \text { for } m \text {-a.e. } x \in \mathbb{R}^{d} \text {. }
$$

The following is now an easy consequence of the results obtained here, in [17] and [15].

Proposition 4.9 Consider the assumptions of Remark 4.5. Then $\left(T_{t}\right)_{t>0}$ (or equivalently $\mathbb{M}$ ) is either transient or recurrent in the sense of [17].

(i) Suppose $\left(T_{t}\right)_{t>0}$ is transient in the sense of [17]. Then for any compact $K \subset \mathbb{R}^{d}$, it holds $\mathbb{P}_{x}\left(L_{K}<\infty\right)=1$ for all $x \in \mathbb{R}^{d}$. In particular

$$
\mathbb{P}_{x}\left(\lim _{t \rightarrow \infty} X_{t}=\Delta \text { in } \mathbb{R}_{\Delta}^{d}\right)=1 \text { for any } x \in \mathbb{R}^{d} .
$$

(ii) Suppose $\left(T_{t}\right)_{t>0}$ is recurrent in the sense of [17]. Then $\mathbb{M}$ is non-explosive and recurrent (in the probabilistic sense), i.e. (62) holds for any nonempty open $U \subset \mathbb{R}^{d}$.

Proof The first assertion follows from Corollary 4.8(i) and [17, Remark 3(b)].

(i) Applying [17, Lemma 6] and the last part of Lemma 3.14(i) we get the existence of $g \in$ $L^{1}\left(\mathbb{R}^{d}, m\right) \cap L^{\infty}\left(\mathbb{R}^{d}, m\right)$ with $g>0$ everywhere, such that $R g:=\mathbb{E}$. $\left[\int_{0}^{\infty} g\left(X_{t}\right) d t\right] \in L^{\infty}\left(\mathbb{R}^{d}, m\right)$. Using that $R g$ is lower semicontinuous by the strong Feller property and essentially bounded, we deduce $R g(x)<\infty$ for any $x \in \mathbb{R}^{d}$. Obviously, $0<R g(x)$ for any $x \in \mathbb{R}^{d}$. Modifying the proof of [17, Proposition 10] (which originates from [15]) with the open sets $U_{n}:=\left\{R g>\frac{1}{n}\right\}, n \geq 1$, and using the strong Feller property of $\left(P_{t}\right)_{t>0}$, we obtain $\mathbb{P}_{x}\left(L_{U_{n}}<\infty\right)=1$ for all $x \in \mathbb{R}^{d}, n \geq 1$. Now the first assertion follows easily since $\left(U_{n}\right)_{n \geq 1}$ is an open cover of any compact set $K \subset \mathbb{R}^{d}$. 
The second assertion follows from the first since the paths of $\mathbb{M}$ are continuous on the one point compactification $\mathbb{R}_{\Delta}^{d}$.

(ii) (48) is a consequence of [17, Corollary 20] and $\mathbb{M}$ is hence non-explosive by (49). Moreover, the right hand side of (63) holds for any $\emptyset \neq U \subset \mathbb{R}^{d}, U$ open, by [17, Proposition 11(d)]. Therefore $\mathbb{M}$ is recurrent in the probabilistic sense.

Remark 4.10 In Proposition 4.9, we get actually equivalences in (i) and (ii). Namely, (64) implies that [17, Condition (8) of Proposition 10] is satisfied. Thus (64) implies transience of $\mathbb{M}$ (or equivalently $\left(T_{t}\right)_{t>0}$ ) in the sense of [17] by [17, Proposition 10]. Likewise, if $\mathbb{M}$ is recurrent (in the probabilistic sense), then it cannot satisfy (64). Therefore, by Proposition 4.9(i) and its first part, $\left(T_{t}\right)_{t>0}$ must be recurrent in the sense of [17].

Define for $r \geq 0$,

$$
v_{1}(r):=\int_{B_{r}} \frac{\langle A(x) x, x\rangle}{\|x\|^{2}} m(d x), \quad v_{2}(r):=\int_{B_{r}}|\langle\mathbf{B}(x), x\rangle| m(d x),
$$

where $\mathbf{B}$ is defined as in Theorem 3.2 and let

$$
v(r):=v_{1}(r)+v_{2}(r), \quad a_{n}:=\int_{1}^{n} \frac{r}{v(r)} d r, \quad n \geq 1 .
$$

Theorem 4.11 (Corollary of [17, Theorem 21]) Consider the assumptions of Remark 4.5] and suppose that

$$
\lim _{n \rightarrow \infty} a_{n}=\infty \quad \text { and } \quad \lim _{n \rightarrow \infty} \frac{\log \left(v_{2}(n) \vee 1\right)}{a_{n}}=0 .
$$

Then $\mathbb{M}$ is recurrent (in the probabilistic sense), non-explosive and $m$ is an invariant measure for $\left(T_{t}\right)_{t \geq 0}$.

Proof By [17, Theorem 21] applied with $\rho(x)=\|x\|$ (the $\rho$ of [17] is different from the $\rho$ defined here), the given assumption implies that $\left(T_{t}\right)_{t>0}$ is not transient in the sense of [17]. Then apply Proposition 4.9. The same considerations apply to $\left(\widehat{T}_{t}\right)_{t>0}$, so that $\left(\widehat{T}_{t}\right)_{t>0}$ is in particular conservative and then $m$ is an invariant measure for $\left(T_{t}\right)_{t \geq 0}$ (and also $\left.\left(\widehat{T}_{t}\right)_{t>0}\right)$.

Lemma 4.12 Consider the assumptions of Remark 4.5. Then for any $x \in \mathbb{R}^{d}$ and $N \in \mathbb{N}$, we have $\mathbb{P}_{x}\left(\sigma_{N}<\infty\right)=1$.

Proof Suppose to the contrary that there exists $N \in \mathbb{N}$ and $x \in \bar{B}_{N}$ such that $\mathbb{P}_{x}\left(\sigma_{N}=\infty\right) \geq$ $\delta>0$. Then $\mathbb{M}$ is not recurrent in the probabilistic sense. Applying Proposition 4.9, we obtain $\mathbb{P}_{x}\left(L_{K}<\infty\right)=1$ for all $x \in \mathbb{R}^{d}$ and any compact $K \subset \mathbb{R}^{d}$. Therefore $\mathbb{P}_{x}\left(\sigma_{N}=\infty\right) \geq \delta>0$ cannot hold and the assertion follows.

The following theorem extends [25, Chapter 6, Theorem 1.2] to locally unbounded drift coefficient. 
Theorem 4.13 Consider the assumptions of Remark 4.5 and suppose that there exists a positive $\psi \in C^{2}\left(\mathbb{R}^{d}\right)$ and some $N_{0} \in \mathbb{N}$ such that $L \psi \leq 0$ a.e. on $\mathbb{R}^{d} \backslash B_{N_{0}}$ and $\inf _{\partial B_{n}} \psi \rightarrow \infty$ as $n \rightarrow \infty$. Then $\mathbb{M}$ of Theorem 3.12 is recurrent (in the probabilistic sense) and non-explosive. In particular, the assumptions above are satisfied (take $\left.\psi(x)=\ln \left(\|x\|^{2}+1\right)+1\right)$, if there is some $N_{0} \in \mathbb{N}$, such that

$$
-\frac{\langle A(x) x, x\rangle}{\|x\|^{2}+1}+\frac{1}{2} \operatorname{trace} A(x)+\langle\mathbf{G}(x), x\rangle \leq 0
$$

for a.e. $x \in \mathbb{R}^{d} \backslash B_{N_{0}}$.

Proof Clearly, $\mathbb{M}$ is non-explosive by Remark 4.3(iii). Let $n \geq N_{0}$ and $x \in \mathbb{R}^{d} \backslash \bar{B}_{n}$ be arbitrary. Choose any $N \in \mathbb{N}$ with $x \in B_{N}$. We will first show that $\mathbb{P}_{x}\left(\sigma_{B_{n}}<\infty\right)=1$. Using that $L \psi \leq 0$ a.e. on $\mathbb{R}^{d} \backslash B_{N_{0}}$ we can see that

$$
\mathbb{E}_{x}\left[\psi\left(X_{t \wedge \sigma_{B n} \wedge \sigma_{N}}\right)\right] \leq \psi(x) .
$$

Since $\mathbb{P}_{x}\left(\sigma_{N}<\infty\right)=1$ by Lemma 4.12, we can let $t \rightarrow \infty$ and obtain with elementary calculations (cf. for instance the proof of Theorem 4.2)

$$
\left(\inf _{\partial B_{N}} \psi\right) \cdot \mathbb{P}_{x}\left(\sigma_{B_{n}}=\infty\right) \leq \mathbb{E}_{x}\left[\psi\left(X_{\sigma_{N}}\right) 1_{\left\{\sigma_{B_{n}}=\infty\right\}}\right] \leq \mathbb{E}_{x}\left[\psi\left(X_{\sigma_{B_{n}} \wedge \sigma_{N}}\right)\right] \leq \psi(x) .
$$

Letting $N \rightarrow \infty$ and using the further assumption on $\psi$, we get $\mathbb{P}_{x}\left(\sigma_{B_{n}}=\infty\right)=0$ and the claim is shown. From now on let $n:=N_{0}+1$. Then obviously $\mathbb{P}_{x}\left(\sigma_{B_{n}}<\infty\right)=1$ for any $x \in B_{n}$ and by the claim $\mathbb{P}_{x}\left(\sigma_{B_{n}}<\infty\right)=1$ for any $\mathbb{R}^{d} \backslash \bar{B}_{n}$. If $x \in \partial B_{n}$, then by the claim again $\mathbb{P}_{x}\left(\sigma_{B_{N_{0}}}<\infty\right)=1$ and since $\sigma_{B_{N_{0}+1}} \leq \sigma_{B_{N_{0}}}$, we finally get

$$
\mathbb{P}_{x}\left(\sigma_{B_{n}}<\infty\right)=1 \quad \text { for any } x \in \mathbb{R}^{d} .
$$

Let $z \in \mathbb{R}^{d}, s>0$ be arbitrary. Then by the Markov property and since $\mathbb{M}$ is non-explosive

$$
\mathbb{P}_{z}\left(X_{t} \in B_{n} \text { for some } t \in[s, \infty)\right)=\mathbb{P}_{z}\left(\sigma_{B_{n}} \circ \vartheta_{s}<\infty\right)=\mathbb{E}_{z}\left[\mathbb{P}_{X_{s}}\left(\sigma_{B_{n}}<\infty\right)\right]=1 \text {. }
$$

Hence $\mathbb{P}_{z}\left(L_{\bar{B}_{N_{0}+1}}<\infty\right)=0$ and the assertion now follows from Proposition 4.9.

\subsubsection{Uniqueness of invariant probability measures and ergodic properties in case $m$ is a probability measure}

In this subsection, we suppose (except at the very end of it) that $m$ is a finite measure. Dividing by a normalizing constant, which will not change the generator $L$, we may without loss of generality assume that $m$ is a probability measure. Coming back to the situation at the beginning of Section 4.2, we have the following:

Remark 4.14 If $m$ is a probability measure, then $m$ is $\left(T_{t}\right)_{t>0}$-invariant, if and only if it is $\left(\widehat{T}_{t}\right)_{t>0}$-invariant (cf. [30, Proposition 1.10(b)]). In either case $\mathbb{P}_{m}$ is then a stationary distribution.

It is clear that the $\left(\widehat{T}_{t}\right)_{t>0}$-invariance of $m$ is equivalent to the conservativeness of $\left(T_{t}\right)_{t>0}$, i.e. to (48). Consequently, using Remark 4.14, we see that $m$ is an invariant (probability) measure for $\left(T_{t}\right)_{t>0}$, if (48) holds. Therefore, (3) provides an explicit criterion for $m$ to be an invariant (probability) measure. Now, we have the following: 
Theorem 4.15 Let $\mathbb{M}$ be as in Theorem 3.12. Suppose that $m$ is a probability measure and that (48) holds. Then:

(i) $m$ is strongly mixing (cf. [26]) and for arbitrary $x \in \mathbb{R}^{d}$ and $A \in \mathcal{B}\left(\mathbb{R}^{d}\right)$

$$
\lim _{t \rightarrow \infty} \mathbb{P}_{x}\left(X_{t} \in A\right)=m(A) .
$$

(ii) $m$ is the unique probability measure that is $\left(P_{t}\right)_{t>0}$-invariant (in the sense of [26]).

(iii) $m$ is equivalent to $\mathbb{P}_{x} \circ X_{t}^{-1}$ for any $(x, t) \in \mathbb{R}^{d} \times(0, \infty)$.

(iv) Let $A \in \mathcal{B}\left(\mathbb{R}^{d}\right)$ be such that $m(A)>0$ and $\left(t_{n}\right)_{n \geq 1} \subset(0, \infty)$ be any sequence with $\lim _{n \rightarrow \infty} t_{n}=\infty$. Then

$$
\mathbb{P}_{x}\left(X_{t_{n}} \in A \text { for infinitely many } n \in \mathbb{N}\right)=1, \quad \forall x \in \mathbb{R}^{d} .
$$

In particular, $\mathbb{M}$ is recurrent.

Proof By Theorem [3.8, Lemma 3.14(i) and Corollary 4.8(ii), $\left(P_{t}\right)_{t>0}$ is strong Feller and $\mathbb{M}$ is irreducible (in the probabilistic sense). Then [26, Proposition 4.1.1] implies that $\left(P_{t}\right)_{t>0}$ is regular. Therefore the assertions (i)-(iii) follow by Doob's Theorem, see [26, Theorem 4.2.1]. Then using (i), assertion (iv) follows by [26, Theorem 3.4.5].

Remark 4.16 Assume that as in Remark 4.5, $\rho$, A, B are explicitly given and that $m=\rho d x$ is a probability measure such that (48) holds. Then Theorem 4.15 applies. This result seems to be new even if $\mathbf{B} \equiv 0$.

For the rest of the section we do not assume that $m$ is a finite measure and present a condition that is independent of $\rho$ and makes Theorem 4.15 applicable. The following proposition is a variant of [25, Chapter 6, Theorem 1.3] which can be applied to locally unbounded drift coefficients.

Proposition 4.17 Under the assumptions of Theorem 3.2, suppose that there exists a positive $\psi \in C^{2}\left(\mathbb{R}^{d}\right)$, some $N_{0} \in \mathbb{N}$ and $C>0$, such that $L \psi \leq-C$ a.e. on $\mathbb{R}^{d} \backslash B_{N_{0}}$ and $\inf _{\partial B_{n}} \psi \rightarrow \infty$ as $n \rightarrow \infty$. Then $m$ is finite and $\mathbb{M}$ is non-explosive. In particular, (48) holds and by normalizing $m$ if necessary, we can see that the assumptions of Theorem 4.15 are satisfied. Thus Theorem 4.15(i)-(iv) hold. In particular, the assumptions above are satisfied (take $\psi(x)=\ln \left(\|x\|^{2}+1\right)+$ 1 ), if there exists a constant $C>0$ and some $N_{0} \in \mathbb{N}$, such that

$$
-\frac{\langle A(x) x, x\rangle}{\|x\|^{2}+1}+\frac{1}{2} \operatorname{trace} A(x)+\langle\mathbf{G}(x), x\rangle \leq-C\left(\|x\|^{2}+1\right)
$$

for a.e. $x \in \mathbb{R}^{d} \backslash B_{N_{0}}$.

Proof Using $L \psi(x) \leq-C$ for a.e. $x \in \mathbb{R}^{d} \backslash B_{N_{0}}$, the finiteness of $m$ follows by [7, Corollary 2.3.3] or [8, Theorem 2] for the original result. Since $L \psi(x) \leq M \psi(x)$ for a.e. $x \in \mathbb{R}^{d} \backslash B_{N_{0}}$ for any $M>0, \mathbb{M}$ is non-explosive by Remark 4.3(iii). We may hence assume that the conditions of Theorem 4.15 are satisfied.

In the next example, we shall give a sufficient condition for (67) to hold. 
Example 4.18 Let $I$ be the identity matrix consisting of ones on the diagonal and zeros outside and set $A(x):=\Psi(x) I$ where $\Psi(x) \in H_{l o c}^{1, p}\left(\mathbb{R}^{d}\right) \cap C_{l o c}^{1-d / p}\left(\mathbb{R}^{d}\right)$ with $\Psi(x)>0$ for all $x \in \mathbb{R}^{d}$. Let $\phi_{1} \in L_{\text {loc }}^{p}\left(\mathbb{R}^{d}\right), \phi_{1} \geq 0$ a.e. and $\mathbf{G}(x):=\left(-\phi_{1}(x) 1_{\mathbb{R}^{d} \backslash B_{N_{0}}}+\phi_{2}(x) 1_{B_{N_{0}}}\right) x$ for some $\phi_{2} \in$ $L_{\text {loc }}^{p}\left(\mathbb{R}^{d}\right)$. Suppose that for some $N_{0} \in \mathbb{N} \cup\{0\}$,

$$
\frac{d}{2} \Psi(x)+C\left(\|x\|^{2}+1\right) \leq \phi_{1}(x)\|x\|^{2} \text { a.e. } x \in \mathbb{R}^{d} \backslash B_{N_{0}} .
$$

Then (68) implies (67).

Now we compare our results with results of [39].

Remark 4.19 As one can see from the proof of Theorem 4.15 in order to derive the conclusions Theorem 4.15(i)-(iv) one needs for instance the classical strong Feller property of $\left(P_{t}\right)_{t>0}$ and the irreducibility of $\mathbb{M}$. In our case, these are directly implied under the conditions of Theorem 3.2 (cf. Theorem 3.8 and Corollary $4.8($ ii)). But the conditions to obtain the strong Feller property and irreducibility in [39] are quite strong, and there are many cases where (67) is satisfied but one cannot obtain the strong Feller property nor irreducibility from the results of [39]. The following provides a comparison of (67) and the rather strong conditions of [39]:

a) If $\mathbf{G}$ is not bounded on an open ball, in order to get the strong Feller property and the irreducibility, [39, Theorem 1.7] needs very strong conditions [39, (H1'), (H2')] such as global uniform ellipticity and boundedness of $A$ and Lipschitz continuity of $A, \mathbf{G}$ and the growth condition $\|\mathbf{G}(x)\| \leq C(1+\|x\|)$ outside an open ball. For example if we take $A(x)=(1+\|x\|) I$ and $\phi_{1}(x)=\|x\|^{2}$, then (68) holds, but (H1') and (H2') in [39] are both not satisfied. Thus the conditions of [39] do neither provide global well-posedness, nor strong Feller properties, nor irreducibility and so on, whereas we get the full conclusions of Proposition 4.17 .

b) If $\mathbf{G}$ is locally bounded on $\mathbb{R}^{d}$, to get the strong Feller property and the irreducibility, [39, Theorem 1.2] also requires quite strong conditions. For example, a diffusion matrix with strong decay such as $A(x)=\exp \left(-\exp \left(\|x\|^{2}\right)\right) I$ cannot be handled by results of [39], since [39, (1.4)] is not satisfied, but we do not have such restrictions. Moreover, if $A(x)=I$ and $\left.\phi_{1}(x)=\exp \left(\exp \left(\|x\|^{2}\right)\right)\right)$, then clearly (68) is satisfied, but [39, (1.7)] is not satisfied. Note that [39, (1.6), (1.8)] requires $A$ to be (besides an $H_{l o c}^{1, q}$-condition, $\left.q>d+2\right)$ locally Lipschitz outside an open ball, if $b \equiv 0$ in [39]), which is also stronger than our condition $a_{i j} \in H_{l o c}^{1, p}\left(\mathbb{R}^{d}\right)$ for $1 \leq i, j \leq d$ for some $p>d$.

(ii) We will give a simple example which has a global pathwise unique solution satisfying all conclusions of Proposition [4.17, but the non-explosion conditions in [39] do even not allow to obtain the existence of global solution. Choose $\Psi(x)=\phi_{1}(x)=(1+\|x\|)^{2}$. Then (68) is satisfied, so that by Example 4.18 we may apply Proposition 4.17 and get a global pathwise unique solution satisfying (i)-(iv) of Theorem 4.15. Now consider

$$
\phi_{2}(x)=\frac{1}{\left\|x-\left(\frac{N_{0}}{2}, 0, \ldots, 0\right)\right\|^{d /(p+1)}}, \quad x \in \mathbb{R}^{d} .
$$

Then $\phi_{2} \in L_{l o c}^{p}\left(\mathbb{R}^{d}\right)$ and $\lim _{x \rightarrow\left(\frac{N_{0}}{2}, 0, \ldots, 0\right)} \phi_{2}(x)=\infty$, so that $\mathbf{G}$ as defined in Example 4.18 satisfies

$$
\langle\mathbf{G}(x), x\rangle \longrightarrow \infty \text { as } x \rightarrow\left(\frac{N_{0}}{2}, 0, \ldots, 0\right) .
$$


Thus, the non-explosion condition [39, (1.5)] is not satisfied and obviously global boundedness of $A$ and linear growth of $\|\mathbf{G}\|$ do not hold, which means [39, [H1'] [H2']] are not satisfied. In particular, no non-explosion condition of [39] holds.

(iii) By our method we have directly a candidate for invariant measure, namely $m$. In [39] no candidate for invariant measure can be deduced.

\section{An application to pathwise uniqueness and strong solutions: the main theorem}

We present here an application of our results to pathwise uniqueness and existence of strong solutions up to $\infty$.

Theorem 5.1 Let $A=\left(a_{i j}\right)_{1 \leq i, j \leq d}, \mathbf{G}$, be as in Theorem [3.2, such that $A=\sigma \sigma^{T}$ and $\sigma=$ $\left(\sigma_{i j}\right)_{1 \leq i, j \leq d}$ satisfies $\sigma_{i j} \in H_{l o c}^{1, p}\left(\mathbb{R}^{d}\right), 1 \leq i, j \leq d$. Suppose that (3) holds for $A$ and $\mathbf{G}$ (or more generally any condition that guarantees the non-explosion of $\mathbb{M}$ of Theorem 3.12). Then the stochastic differential equation

$$
X_{t}=x_{0}+\int_{0}^{t} \sigma\left(X_{s}\right) d W_{s}+\int_{0}^{t} \mathbf{G}\left(X_{s}\right) d s, 0 \leq t<\infty, x_{0} \in \mathbb{R}^{d},
$$

where $W=\left(W^{1}, \ldots, W^{d}\right)$ is a standard d-dimensional Brownian motion starting from zero, has a pathwise unique and strong solution $\left(X_{t}\right)_{t \geq 0}$. In particular, and without any further assumption, $\left(X_{t}\right)_{t \geq 0}$ satisfies more than classical strong Feller properties (see Theorem [3.8, Proposition 3.10 and Lemma 3.14(i)), has integrability properties as in Lemma 3.15, is irreducible (by Corollary 4.8), satisfies the long time behavior as in Proposition 4.9 and Remark 4.10, and has further additional properties like in Lemma 4.6. Remark 4.7, Lemma 4.12. Moreover, there are diverse explicit further conditions to guarantee moment inequalities, recurrence and ergodicity, including existence and uniqueness of invariant probability measures for $\left(X_{t}\right)_{t \geq 0}$, see Theorems 4.4. 4.11. 4.13 and Proposition 4.17 (or for the more general statement see Theorem 4.15). Finally, Lemma 3.14 (ii) also holds for $\left(X_{t}\right)_{t \geq 0}$.

Proof The existence of a weak solution up to $\zeta=\infty$ under the present assumptions follows from Theorems 3.19 (i) and 4.2. This then leads to the existence of a pathwise unique and strong global solution by [38, Theorem 1.3] and [20, Chapter IV.1. Theorem 1.1]. For all other statements, we refer to the corresponding proofs and note that uniqueness in law is implied by pathwise uniqueness (see for instance [20, Chapter IV.1. Corollary]), so any solution to (69) has the same law.

\section{References}

[1] S. Albeverio, Yu. G. Kondratiev, M. Röckner, Strong Feller properties for distorted Brownian motion and applications to finite particle systems with singular interactions, Finite and infinite dimensional analysis in honor of Leonard Gross (New Orleans, LA, 2001), 15-35, Contemp. Math., 317, Amer. Math. Soc., Providence, RI, 2003. 
[2] D. G. Aronson, J. Serrin, Local behavior of solutions of quasilinear parabolic equations, Arch. Rational Mech. Anal. 25 (1967), 81-122.

[3] K. Bahlali, Flows of homeomorphisms of stochastic differential equations with measurable drift, Stochastics Stochastics Rep. 67 (1999), no. 1-2, 53-82.

[4] R. N. Bhattacharya, Criteria for recurrence and existence of invariant measures for multidimensional diffusions, Ann. Probab. 6 (1978), no. 4, 541-553.

[5] B. Baur, M. Grothaus, P. Stilgenbauer, Construction of $\mathcal{L}^{p}$ - strong Feller processes via Dirichlet forms and applications to elliptic diffusions, Potential Anal. 38 (2013), no. 4, $1233-1258$.

[6] V. I. Bogachev, N. Krylov, M. Röckner, On regularity of transition probabilities and invariant measures of singular diffusions under minimal conditions, Comm. Partial Differential Equations 26 (2001), no. 11-12, 2037-2080.

[7] V. I. Bogachev, N. V. Krylov, M. Röckner, and S. V. Shaposhnikov, Fokker-PlanckKolmogorov equations, Mathematical Surveys and Monographs, 207. American Mathematical Society, Providence, RI, 2015.

[8] V. I. Bogachev, M. Röckner, and S. V. Shaposhnikov, On positive and probability solutions of the stationary Fokker-Planck-Kolmogorov equation. (Russian) Dokl. Akad. Nauk 444 (2012), no. 3, 245-249; translation in Dokl. Math. 85 (2012), no. 3, 350-354.

[9] Z. Chen, Z. Huan, On the continuity of the mth root of a continuous nonnegative definite matrix-valued function, J. Math. Anal. Appl. 209 (1997), no. 1, 6066.

[10] K. L. Chung, J. B. Walsh, Markov processes, Brownian motion, and time symmetry, Second edition. Grundlehren der Mathematischen Wissenschaften, 249. Springer, New York, 2005.

[11] E.B. Davies, $L^{1}$ properties of second order elliptic operators, Bull. London Math. Soc. 17 (1985), no. 5, 417-436.

[12] R. Durrett, Stochastic calculus. A practical introduction. Probability and Stochastics Series. CRC Press, Boca Raton, FL, 1996.

[13] E. Fedrizzi, F. Flandoli, Pathwise uniqueness and continuous dependence of SDEs with non-regular drift, Stochastics 83 (2011), no. 3, 241-257.

[14] E. Fedrizzi, F. Flandoli, Hölder flow and differentiability for SDEs with nonregular drift, Stoch. Anal. Appl. 31 (2013), no. 4, 708-736.

[15] R. K. Getoor, Transience and recurrence of Markov processes. Seminar on Probability. XIV. pp. 397-409. Lecture Notes in Math. 784, Springer. Berlin. 1980.

[16] M. Gim, G. Trutnau, Conservativeness criteria for generalized Dirichlet forms Journal of Mathematical Analysis and Applications, Volume 448, Issue 2, (2017), pp. 1419-1449

[17] M. Gim, G. Trutnau, Recurrence criteria for generalized Dirichlet forms, J. Theoret. Probab. 31 (2018), no. 4, 2129-2166, arXiv:1508.02282. 
[18] I. Gyöngy, T. Martinez, On stochastic differential equations with locally unbounded drift, Czechoslovak Math. J. (4) 51 (126) (2001) 763-783.

[19] R. Z. Has'minskii, Ergodic properties of recurrent diffusion processes and stabilization of the solution of the Cauchy problem for parabolic equations, Teor. Verojatnost. i Primenen. 5, 1960, 196214.

[20] N. Ikeda, S. Watanabe, Stochastic differential equations and diffusion processes, Second edition. North-Holland Mathematical Library, 24. North-Holland Publishing Co., Amsterdam; Kodansha, Ltd., Tokyo, 1989.

[21] N. V. Krylov, M. Röckner, Strong solutions for stochastic equations with singular time dependent drift, Prob. Th. Rel. Fields 131 (2005), no. 2, 154-196.

[22] X. Mao, Stochastic differential equations and applications, Second edition. Horwood Publishing Limited, Chichester, 2008.

[23] C. B. Jr. Morrey, Multiple integrals in the calculus of variations, Reprint of the 1966 edition, Classics in Mathematics. Springer-Verlag, Berlin, 2008.

[24] A. Pazy, Semigroups of linear operators and applications to partial differential equations, Applied Mathematical Sciences, 44. Springer-Verlag, New York, 1983.

[25] R. G. Pinsky Positive Harmonic Functions and Diffusion, Cambridge Studies in Advanced Mathematics 45. Cambridge University Press. Cambridge. 1995.

[26] G. Da Prato, J. Zabczyk, Ergodicity for infinite-dimensional systems, London Mathematical Society Lecture Note Series, 229. Cambridge University Press, Cambridge, 1996.

[27] M. Röckner, J. Shin, G. Trutnau, Non-symmetric distorted Brownian motion: strong solutions, strong Feller property and non-explosion results, Discrete Contin. Dyn. Syst. Ser. B 21 (2016), no. 9, 3219-3237.

[28] J. Shin, G. Trutnau, On the stochastic regularity of distorted Brownian motions, Trans. Amer. Math. Soc. 369 (2017), pp. 7883-7915, arXiv:1405.7585.

[29] W. Stannat, The Theory of Generalized Dirichlet Forms and Its Applications in Analysis and Stochastics. Dissertation, Bielefeld 1996. Published as Memoirs of the AMS. Volume 142. No. 678. 1999.

[30] W. Stannat, (Nonsymmetric) Dirichlet operators on $L^{1}$ : Existence, uniqueness and associated Markov processes, Ann. Scuola Norm. Sup. Pisa Cl. Sci. (4) 28. 1999. No. 1. 99-140.

[31] Daniel W. Stroock, S. R. Srinivasa Varadhan, Multidimensional diffusion processes Reprint of the 1979 edition. Classics in Mathematics. Springer-Verlag, Berlin, 2006.

[32] N. S. Trudinger, Linear elliptic operators with measurable coefficients, Ann. Scuola Norm. Sup. Pisa (3) 27 (1973), 265-308.

[33] G. Trutnau, On Hunt processes and strict capacities associated with generalized Dirichlet forms, Infin. Dimens. Anal. Quantum Probab. Relat. Top. 8 (2005), no. 3, 357-382. 
[34] G. Trutnau, On a class of non-symmetric diffusions containing fully non-symmetric distorted Brownian motions, Forum Math. 15(3), 409-437 (2003).

[35] A. Yu. Veretennikov, On the strong solutions of stochastic differential equations, Theory Probab. Appl. 24 (1979) 354-366.

[36] Yu.A. Veretennikov, On strong solution and explicit formulas for solutions of stochastic integral equations, Math. USSR Sb. 39, 387403 (1981).

[37] X. Zhang, Strong solutions of SDES with singular drift and Sobolev diffusion coefficients, Stochastic Process. Appl. 115 (2005), no. 11, 1805-1818.

[38] X. Zhang, Stochastic homeomorphism flows of SDEs with singular drifts and Sobolev diffusion coefficients, Electron. J. Probab. 16 (2011), no. 38, 1096-1116.

[39] L. Xie, X. Zhang, Sobolev differentiable flows of SDEs with local Sobolev and super-linear growth coefficients, Ann. Probab. 44 (2016), no. 6, 3661-3687

[40] A. K. Zvonkin, A transformation of the phase space of a diffusion process that will remove the drift, Mat. Sb. (N.S.) 93(135) (1974), 129-149.

Haesung Lee, Gerald Trutnau

Department of Mathematical Sciences and

Research Institute of Mathematics of Seoul National University,

1 Gwanak-Ro, Gwanak-Gu, Seoul 08826, South Korea,

E-mail: fthslt@snu.ac.kr, trutnau@snu.ac.kr 